\title{
UNCONDITIONAL CONVERGENCE OF SPECTRAL DECOMPOSITIONS OF 1D DIRAC OPERATORS WITH REGULAR BOUNDARY CONDITIONS
}

\author{
PLAMEN DJAKOV AND BORIS MITYAGIN
}

Abstract. One dimensional Dirac operators

$$
L_{b c}(v) y=i\left(\begin{array}{cc}
1 & 0 \\
0 & -1
\end{array}\right) \frac{d y}{d x}+v(x) y, \quad y=\left(\begin{array}{l}
y_{1} \\
y_{2}
\end{array}\right), \quad x \in[0, \pi],
$$

considered with $L^{2}$-potentials $v(x)=\left(\begin{array}{cc}0 & P(x) \\ Q(x) & 0\end{array}\right)$ and subject to regular boundary conditions $(b c)$, have discrete spectrum.

For strictly regular $b c$, it is shown that every eigenvalue of the free operator $L_{b c}^{0}$ is simple and has the form $\lambda_{k, \alpha}^{0}=k+\tau_{\alpha}$ where $\alpha \in\{1,2\}, k \in 2 \mathbb{Z}$ and $\tau_{\alpha}=\tau_{\alpha}(b c)$; if $|k|>N(v, b c)$ each of the $\operatorname{discs} D_{k}^{\alpha}=\left\{z:\left|z-\lambda_{k, \alpha}^{0}\right|<\rho=\rho(b c)\right\}, \alpha \in\{1,2\}$, contains exactly one simple eigenvalue $\lambda_{k, \alpha}$ of $L_{b c}(v)$ and $\left(\lambda_{k, \alpha}-\lambda_{k, \alpha}^{0}\right)_{k \in 2 \mathbb{Z}}$ is an $\ell^{2}$-sequence. Moreover, it is proven that the root projections $P_{n, \alpha}=\frac{1}{2 \pi i} \int_{\partial D_{n}^{\alpha}}\left(z-L_{b c}(v)\right)^{-1} d z$ satisfy the Bari-Markus condition

$$
\sum_{|n|>N}\left\|P_{n, \alpha}-P_{n, \alpha}^{0}\right\|^{2}<\infty, \quad n \in 2 \mathbb{Z},
$$

where $P_{n}^{0}$ are the root projections of the free operator $L_{b c}^{0}$. Hence, for strictly regular $b c$, there is a Riesz basis consisting of root functions (all but finitely many being eigenfunctions). Similar results are obtained for regular but not strictly regular $b c$ - then in general there is no Riesz basis consisting of root functions but we prove that the corresponding system of two-dimensional root projections is a Riesz basis of projections.

Keywords: Dirac operators, Riesz bases, regular boundary conditions MSC: 47E05, 34L40, 34L10.

\section{CONTENT}

1. Introduction

2. Technical preliminaries; Riesz systems of projections

3. General regular and strictly regular boundary conditions

This paper was completed at the Mathematisches Forschungsinstitut Oberwolfach during our three week stay there in August 2010 within the Research in Pairs Programme. We appreciate the hospitality and creative atmosphere of the Institute. 
4. Matrix representation of $L_{b c}$ and its resolvent $R_{b c}(\lambda)$

5. Localization of spectra

6. Bari-Markus property in the case of strictly regular boundary conditions

7. Bari-Markus property in the case of regular but not strictly regular boundary conditions

8. Miscellaneous; pointwise convergence and equiconvergence

\section{INTRODUCTION}

Spectral theory of non-selfadjoint boundary value problems $(B V P)$ for ordinary differential equations on a finite interval $I$ goes back to the classical works of Birkhoff [2, 3] and Tamarkin [26, 27, 28]. They introduced a concept of regular $(R)$ boundary conditions $(b c)$ and investigated asymptotic behavior of eigenvalues and eigenfunctions of such problems. Moreover, they proved that the system of eigenfunctions and associated functions $(S E A F)$ of a regular $B V P$ is complete.

More subtle is the question whether $S E A F$ is a basis or an unconditional basis in the Hilbert space $H^{0}=L^{2}(I)$. N. Dunford [11] (see also [12]), V. P. Mikhailov [18], G. M. Keselman [15] independently proved that the $S E A F$ is an unconditional, or Riesz, basis if $b c$ are strictly regular $(S R)$. This property is lost if $b c$ are $R \backslash S R$, i.e., regular but not strictly regular; unfortunately, this is just the case of periodic $\left(\mathrm{Per}^{+}\right)$ and anti-periodic $\left(\mathrm{Per}^{-}\right)$bc. But A. A. Shkalikov [22, 23, 24] proved that in $R \backslash S R$ cases a proper chosen finite-dimensional projections form a Riesz basis of projections.

Dirac operators

$$
L y=i\left(\begin{array}{cc}
1 & 0 \\
0 & -1
\end{array}\right) \frac{d Y}{d x}+v(x) Y, \quad Y=\left(\begin{array}{l}
y_{1} \\
y_{2}
\end{array}\right), \quad v(x)=\left(\begin{array}{cc}
0 & P(x) \\
Q(x) & 0
\end{array}\right)
$$

with $P, Q \in L^{2}(I)$, and more general operators

$$
M y=i B \frac{d Y}{d x}+v(x) Y, \quad Y=\left(y_{j}(x)\right)_{1}^{d},
$$

where $B$ is a $d \times d$-matrix and $v(x)$ is a $d \times d$ matrix-valued $L^{2}(I)$ function bring new difficulties. One of them comes from the fact that the values of the resolvent $\left(\lambda-L_{b c}\right)^{-1}$ are not trace class operators.

For general system (1.2) M. M. Malamud and L. L. Oridoroga [16] gave sufficient conditions for the completeness and minimality of the $S E A F$ in the case of regular $B V P$.

The Riesz basis property for $2 \times 2$ Dirac operators (1.1) was proved by I. Trooshin and M. Yamamoto [29, 30] in the case of separated $b c$ 
and $v \in L^{2}$. S. Hassi and L. L. Oridoroga [14] proved the Riesz basis

property for (1.2) when $B=\left(\begin{array}{cc}a & 0 \\ 0 & -b\end{array}\right)$, with $a, b>0$, for separated $b c$ and $v \in C^{1}(I)$.

B. Mityagin [19], [20, Theorem 8.8] proved that periodic (or antiperiodic) $b c$ give a rise of a Riesz system of $2 \mathrm{D}$ projections (or $2 \mathrm{D}$ invariant subspaces) under the smoothness restriction $P, Q \in H^{\alpha}, \alpha>$ $1 / 2$, on the potentials $v$ in (1.1). The authors removed that restriction in [9, where the same result is obtained for any $L^{2}$ potential $v$. This became possible in the framework of the general approach to analysis of invariant (Riesz) subspaces and their closeness to 2D subspaces of the free operator developed and used by the authors in $[4,5,6,7,8]$.

Now we extend these results to Dirac operators with any regular bc, which requires a careful analysis of regular and strictly regular (a la Birkhoff-Tamarkin) $b c$ themselves - Section 3 describes these $b c$ and give explicit form of the $S E A F$ (Lemmas 5,6,7) for $\mathrm{SR}$ and $R \backslash$ $S R b c$ in the case of the free Dirac operator. Section 2 reminds the elementary geometry of Riesz bases or Riesz systems of projections in a Hilbert space (see [1, 17, 13]). In Section 4 and 5 we study the analytic properties of the resolvent $R_{b c}(\lambda)=\left(\lambda-L_{b c}\right)^{-1}$ with $v \in L^{2} . S R$ and $R \backslash S R$ cases differ in some technical details, and Theorems 12 and 14 accordingly take care about localization of $L_{b c}$ 's spectra. Now (Sections $6,7)$ the representation of projections as Cauchy-Riesz integrals of the resolvent is used to get Bari-Markus property of the Riesz system for $L_{b c}$. In the $S R$ case this leads (Theorem 15 ) to Riesz basis property of the $S E A F$; in the $R \backslash S R$ case the system of 2D projections of root subspaces is a Riesz system (Theorem 20).

\section{Technical PReliminaries about Riesz Systems of PROJECTIONS}

Here we recall some basic facts about Hilbert-Schmidt operators, Riesz bases, etc. All Hilbert spaces that we consider are supposed to be separable.

1. Hilbert-Schmidt operators.

Let $H$ be a Hilbert space. A linear operator $T: H \rightarrow H$ is HilbertSchmidt operator if its Hilbert-Schmidt norm $\|T\|_{H S}$ is finite, where

$$
\|T\|_{H S}^{2}:=\sum_{\gamma \in \Gamma}\left\|T e_{\gamma}\right\|^{2}=\sum_{\gamma, \beta \in \Gamma}\left|\left\langle T e_{\gamma}, e_{\beta}\right\rangle\right|^{2}
$$


with $\left(e_{\gamma}, \gamma \in \Gamma\right)$ being any orthonormal basis (o.n.b.) in $H$. The following lemma summarizes some of the properties of Hilbert-Schmidt operators and Hilbert-Schmidt norm.

Lemma 1. Let $T: H \rightarrow H$ and $S: H \rightarrow H$ be linear operators.

(a) $\|T\|_{H S}$ in (2.1) does not depend on the choice of o.n.b. $\left(e_{\gamma}\right)$.

(b) $\|T\|_{H S}$ is a norm such that $\left\|T^{*}\right\|_{H S}=\|T\|_{H S}$.

(c) $\|T\| \leq\|T\|_{H S}$

(d) If $T$ is Hilbert-Schmidt and $S$ is bounded, then $S T$ and TS are Hilbert-Schmidt operators, and

$$
\|S T\|_{H S},\|T S\|_{H S} \leq\|T\|_{H S} \cdot\|S\|
$$

(e) Every Hilbert-Schmidt operator is compact.

We refer to [13, 21] for proofs of these properties and more details about Hilbert-Schmidt operators.

2. Riesz bases.

Let $H$ be a Hilbert space, and let $\left(e_{\gamma}, \gamma \in \Gamma\right)$ be an o.n.b. in $H$. If $A: H \rightarrow H$ is an automorphism, then the system

$$
f_{\gamma}=A e_{\gamma}, \quad \gamma \in \Gamma
$$

is an unconditional basis in $H$. Indeed, for each $x \in H$ we have

$$
\begin{gathered}
x=A\left(A^{-1} x\right)=A\left(\sum_{\gamma}\left\langle A^{-1} x, e_{\gamma}\right\rangle e_{\gamma}\right) \\
=\sum_{\gamma}\left\langle x,\left(A^{-1}\right)^{*} e_{\gamma}\right\rangle f_{\gamma}=\sum_{\gamma}\left\langle x, \tilde{f}_{\gamma}\right\rangle f_{\gamma},
\end{gathered}
$$

so $\left(f_{\gamma}\right)$ is a basis, and its biorthogonal system is

$$
\tilde{f}_{\gamma}=\left(A^{-1}\right)^{*} e_{\gamma}, \quad \gamma \in \Gamma .
$$

Moreover, it follows that

$$
0<c \leq\left\|f_{\gamma}\right\| \leq C, \quad m^{2}\|x\|^{2} \leq \sum_{\gamma}\left|\left\langle x, \tilde{f}_{\gamma}\right\rangle\right|^{2}\left\|f_{\gamma}\right\|^{2} \leq M^{2}\|x\|^{2},
$$

with $c=1 /\left\|A^{-1}\right\|, C=\|A\|, M=\|A\| \cdot\left\|A^{-1}\right\|$ and $m=1 / M$.

A basis of the form (2.2) is called Riesz basis. One can easily see that the property (2.4) characterizes Riesz bases, i.e., a basis $\left(f_{\gamma}\right)$ is a Riesz bases if and only if (2.4) holds with some constants $C \geq c>0$ and $M \geq m>0$. Another characterization of Riesz bases gives the following assertion (see [13, Chapter 6, Section 5.3, Theorem 5.2]): If $\left(f_{\gamma}\right)$ is a normalized basis (i.e., $\left\|f_{\gamma}\right\|=1 \forall \gamma$ ), then it is a Riesz basis if and only if it is unconditional. 
Let $\left(f_{\gamma}\right)$ be a fixed Riesz basis in $H$ of the form (2.2). For each Hilbert-Schmidt operator $T$ we consider

$$
\|T\|_{H S}^{*}=\left(\sum_{\gamma, \beta}\left|\left\langle T f_{\gamma}, \tilde{f}_{\beta}\right\rangle\right|^{2}\right)^{1 / 2} .
$$

Then $\|T\|_{H S}^{*}$ is a norm which is equivalent to $\|T\|_{H S}$. Indeed, in view of (2.1) $-(2.3)$,

$$
\begin{aligned}
& \left(\|T\|_{H S}^{*}\right)^{2}=\sum_{\gamma, \beta}\left|\left\langle T A e_{\gamma},\left(A^{-1}\right)^{*} e_{\beta}\right\rangle\right|^{2} \\
= & \sum_{\gamma, \beta}\left|\left\langle A^{-1} T A e_{\gamma}, e_{\beta}\right\rangle\right|^{2}=\left\|A^{-1} T A\right\|_{H S}^{2} .
\end{aligned}
$$

Therefore, in view of Lemma 1,

$$
\|T\|_{H S}^{*}=\left\|A^{-1} T A\right\|_{H S} \leq M\|T\|_{H S}
$$

with $M=\|A\| \cdot\left\|A^{-1}\right\|$. On the other hand, by the same argument,

$$
\|T\|_{H S}=\left\|A\left(A^{-1} T A\right) A^{-1}\right\|_{H S} \leq M\left\|A^{-1} T A\right\|_{H S}=M\|T\|_{H S}^{*} .
$$

3. Riesz bases of projections and Bari-Markus Theorem

Let $H$ be a Hilbert space. A family of bounded finite-dimensional projections $\left\{P_{\gamma}: H \rightarrow H, \gamma \in \Gamma\right\}$ is called unconditional basis of projections if the following conditions hold:

$$
\begin{array}{r}
P_{\alpha} P_{\beta}=0 \quad \text { if } \alpha \neq \beta, \quad P_{\alpha}^{2}=P_{\alpha} ; \\
x=\sum_{\gamma \in \Gamma} P_{\gamma}(x) \quad \forall x \in H,
\end{array}
$$

where the series converge unconditionally.

Obviously, if $\left(f_{\gamma}\right)$ is an unconditionsl basis in $H$ then the system of one-dimensional projections $P_{\gamma}(x)=\tilde{f}_{\gamma}(x) f_{\gamma}$ is a basis of projections in $H$, and vice versa, every basis of one dimensional projections can be obtained in that way from some basis.

If $\left(Q_{\gamma}\right)$ is a basis of orthogonal projections (i.e., $Q_{\gamma}^{*}=Q_{\gamma}$ ), the Pythagorian theorem implies $\sum_{\gamma}\left\|Q_{\gamma} x\right\|^{2}=\|x\|^{2}$.

We say that the family of projections $\left(P_{\gamma}^{0}, \gamma \in \Gamma\right)$ is a Riesz basis of projections if

$$
P_{\gamma}^{0}=A Q_{\gamma} A^{-1}, \quad \gamma \in \Gamma,
$$

where $A: H \rightarrow H$ is an isomorphism and $\left(Q_{\gamma}, \gamma \in \Gamma\right)$ is a basis of orthogonal projections. 
If (2.8) holds, then

$$
\sum_{\gamma}\left\|P_{\gamma}^{0} x\right\|^{2} \leq\|A\|^{2} \sum_{\gamma}\left\|Q_{\gamma} A^{-1} x\right\|^{2}=\|A\|^{2}\left\|A^{-1} x\right\|^{2} \leq M^{2}\|x\|^{2}
$$

with $M=\|A\|\left\|A^{-1}\right\|$.

The following statement is a version of the Bari-Markus theorem (see [13], Ch.6, Sect. 5.3, Theorem 5.2).

Theorem 2. Suppose that $\left(P_{\gamma}, \gamma \in \Gamma\right)$ is a family of bounded finite dimensional projections in a Hilbert space $H$ such that

$$
P_{\alpha} P_{\beta}=0 \quad \text { if } \alpha \neq \beta \text {. }
$$

If there is a Riesz basis of projections $\left(P_{\gamma}^{0}, \gamma \in \Gamma\right)$ such that

$$
\operatorname{dim} P_{\gamma}=\operatorname{dim} P_{\gamma}^{0}, \quad \gamma \in \Gamma,
$$

and

$$
\sum_{\gamma \in \Gamma}\left\|P_{\gamma}-P_{\gamma}^{0}\right\|^{2}<\infty
$$

then $\left(P_{\gamma}\right)$ is a Riesz basis of projections in $H$.

Proof. Let the projections $P_{\gamma}^{0}$ be given by (2.8). In view of (2.12), there is a finite subset $\Gamma_{1} \subset \Gamma$ such that

$$
\sum_{\Gamma \backslash \Gamma_{1}}\left\|P_{\gamma}-P_{\gamma}^{0}\right\|^{2} M^{2}<\frac{1}{4}
$$

where the constant $M=\left\|A^{-1}\right\|\|A\|$ comes from (2.9). Consider the operators

$$
T x=\sum_{\Gamma \backslash \Gamma_{1}}\left(P_{\gamma}-P_{\gamma}^{0}\right) P_{\gamma}^{0} x, \quad B x=\sum_{\Gamma_{1}} P_{\gamma}^{0} x+\sum_{\Gamma \backslash \Gamma_{1}} P_{\gamma} P_{\gamma}^{0} x=x+T x .
$$

In view of (2.9) and (2.13), the Cauchy inequality yields

$$
\|T x\|^{2} \leq\left(\sum_{\Gamma \backslash \Gamma_{1}}\left\|P_{\gamma}-P_{\gamma}^{0}\right\|\left\|P_{\gamma}^{0} x\right\|\right)^{2} \leq \sum_{\Gamma \backslash \Gamma_{1}}\left\|P_{\gamma}-P_{\gamma}^{0}\right\|^{2} \sum_{\Gamma \backslash \Gamma_{1}}\left\|P_{\gamma}^{0} x\right\|^{2} \leq \frac{1}{2}\|x\|^{2} .
$$

Therefore $\|T\|<1 / 2$, which implies that $B: H \rightarrow H$ is an isomorphism.

By the construction of the operator $B$, if $\alpha \in \Gamma \backslash \Gamma_{1}$ then $B$ coincides on the subspace $P_{\alpha}^{0}(H)$ with the projection $P_{\alpha}$, i.e.,

$$
B P_{\alpha}^{0} x=P_{\alpha} P_{\alpha}^{0} x \quad \text { for } \quad \alpha \in \Gamma \backslash \Gamma_{1}, \quad x \in H .
$$


Since $\operatorname{dim} P_{\alpha}^{0}(H)=\operatorname{dim} P_{\alpha}(H)<\infty$, it follows that $B$ maps $P_{\alpha}^{0}(H)$ onto $P_{\alpha}(H)$, which yields $P_{\alpha}=B P_{\alpha}^{0} B^{-1}$ for $\alpha \in \Gamma \backslash \Gamma_{1}$.

Let $H_{1}^{0}, H_{2}^{0}, H_{1}, H_{2}$ be, respectively, the closed linear spans of

$$
\bigcup_{\Gamma_{1}} P_{\gamma}^{0}(H), \quad \bigcup_{\Gamma \backslash \Gamma_{1}} P_{\gamma}^{0}(H), \quad \bigcup_{\Gamma_{1}} P_{\gamma}(H), \quad \bigcup_{\Gamma \backslash \Gamma_{1}} P_{\gamma}(H) ;
$$

then $H=H_{1}^{0} \oplus H_{2}^{0}=H_{1} \oplus H_{2}$, and $B\left(H_{2}^{0}\right)=H_{2}$. Since $\operatorname{dim} P_{\gamma}^{0}(H)=$ $\operatorname{dim} P_{\gamma}(H)$, there exists an isomorphism $\tilde{B}: H \rightarrow H$ such that $\tilde{B}=B$ on $H_{2}^{0}$ and $\tilde{B}$ maps $P_{\gamma}^{0}(H)$ onto $P_{\gamma}(H)$ for every $\gamma$. Thus, by (2.8) we obtain

$$
P_{\gamma}=\tilde{B} P_{\gamma}^{0} \tilde{B}^{-1}=\tilde{B} A Q_{\gamma} A^{-1} \tilde{B}^{-1}=(\tilde{B} A) Q_{\gamma}(\tilde{B} A)^{-1}, \quad \gamma \in \Gamma,
$$

which proves that $\left(P_{\gamma}\right)$ is a Riesz basis of projections.

\section{General Regular and Strictly Regular BOUndary CONDITIONS}

We consider the Dirac operators $L=L(v)$ given by (1.1) on the interval $I=[0, \pi]$ and set $L^{0}=L(0)$. In the following, the Hilbert space $L^{2}\left(I, \mathbb{C}^{2}\right)$ is regarded equipped with the scalar product

$$
\left\langle\left(\begin{array}{l}
f_{1} \\
f_{2}
\end{array}\right),\left(\begin{array}{l}
g_{1} \\
g_{2}
\end{array}\right)\right\rangle=\frac{1}{\pi} \int_{0}^{\pi}\left(f_{1}(x) \overline{g_{1}(x)}+f_{2}(x) \overline{g_{2}(x)}\right) d x .
$$

1. A general boundary condition for the operator $L^{0}$ (or $L$ ) is given by a system of two linear equations

$$
\begin{aligned}
& a_{1} y_{1}(0)+b_{1} y_{1}(\pi)+a_{2} y_{2}(0)+b_{2} y_{2}(\pi)=0 \\
& c_{1} y_{1}(0)+d_{1} y_{1}(\pi)+c_{2} y_{2}(0)+d_{2} y_{2}(\pi)=0
\end{aligned}
$$

Let $A_{i j}$ denote the $2 \times 2$ matrix formed by the $i$-th and $j$-th columns of the matrix

$$
\left[\begin{array}{llll}
a_{1} & b_{1} & a_{2} & b_{2} \\
c_{1} & d_{1} & c_{2} & d_{2}
\end{array}\right]
$$

and let $\left|A_{i j}\right|$ denote the determinant of the matrix $A_{i j}$. Each solution of the equation

$$
L^{0} y=\lambda y, \quad y=\left(\begin{array}{l}
y_{1} \\
y_{2}
\end{array}\right)
$$

has the form

$$
y=\left(\begin{array}{c}
\xi e^{-i \lambda x} \\
\eta e^{i \lambda x}
\end{array}\right)
$$


It satisfies the boundary condition (3.2) if and only if $(\xi, \eta)$ is a solution of the system of two linear equations

$$
\begin{aligned}
& \xi\left(a_{1}+b_{1} z^{-1}\right)+\eta\left(a_{2}+b_{2} z\right)=0 \\
& \xi\left(c_{1}+d_{1} z^{-1}\right)+\eta\left(c_{2}+d_{2} z\right)=0
\end{aligned}
$$

where $z=\exp (i \pi \lambda)$. Therefore, we have a non-zero solution $y$ if and only if the determinant of (3.6) is zero, which is equivalent to the quadratic equation

$$
\left|A_{14}\right| z^{2}+\left(\left|A_{13}\right|+\left|A_{24}\right|\right) z+\left|A_{23}\right|=0 .
$$

Definition 3. The boundary condition (3.2) is called: regular if

$$
\left|A_{14}\right| \neq 0, \quad\left|A_{23}\right| \neq 0,
$$

and strictly regular if additionally

$$
\left(\left|A_{13}\right|+\left|A_{24}\right|\right)^{2} \neq 4\left|A_{14}\right|\left|A_{23}\right|
$$

holds.

Of course, (3.9) is equivalent to saying that the quadratic equation (3.7) has two distinct roots.

From now on we consider only regular boundary conditions. We multiply from the left the system (3.2) and the $2 \times 4$ matrix (3.3) by the matrix $A_{14}^{-1}$. This gives us an equivalent to (3.2) system

$$
\begin{aligned}
& y_{1}(0)+b y_{1}(\pi)+a y_{2}(0)=0 \\
& d y_{1}(\pi)+c y_{2}(0)+y_{2}(\pi)=0
\end{aligned}
$$

which matrix has the form

$$
\left[\begin{array}{llll}
1 & b & a & 0 \\
0 & d & c & 1
\end{array}\right]
$$

and $\left[\begin{array}{cc}b & a \\ d & c\end{array}\right]=A_{14}^{-1} A_{23}$.

In the following we consider only boundary conditions in the form (3.10) with matrices (3.11). Then

$$
\left|A_{14}\right|=1, \quad\left|A_{13}\right|=c, \quad\left|A_{24}\right|=b, \quad\left|A_{23}\right|=b c-a d .
$$

Condition (3.8) means that

$$
\left|A_{23}\right|=b c-a d \neq 0,
$$

and (3.9) takes the form

$$
(b-c)^{2}+4 a d \neq 0 .
$$


Now the system (3.6) becomes

$$
\begin{aligned}
& \xi\left(1+b z^{-1}\right)+\eta a=0 \\
& \xi d z^{-1}+\eta(c+z)=0
\end{aligned}
$$

and the equation (3.7) becomes

$$
z^{2}+(b+c) z+b c-a d=0
$$

Notice that (3.15) means

$$
\left[\begin{array}{cc}
1+b / z & a \\
d / z & c+z
\end{array}\right]\left(\begin{array}{l}
\xi \\
\eta
\end{array}\right)=\left[\begin{array}{cc}
z+b & a \\
d & c+z
\end{array}\right]\left(\begin{array}{c}
\xi / z \\
\eta
\end{array}\right)=0 .
$$

From here (or, since the change of variable $z \rightarrow-w$ transforms (3.16) into the characteristic equation of the matrix $A_{23}$ ) we get the following.

Lemma 4. The number $z$ is a root of (3.16) if and only if $-z$ is an eigenvalue of the matrix $A_{23}=\left[\begin{array}{cc}b & a \\ d & c\end{array}\right]$. Moreover, $\left(\begin{array}{l}\xi \\ \eta\end{array}\right)$ is a non-zero solution of (3.15) if and only if $-z$ is an eigenvalue of the matrix $A_{23}$ and $\left(\begin{array}{c}\xi / z \\ \eta\end{array}\right)$ is an eigenvector of $A_{23}$ corresponding to $-z$.

2. Strictly Regular boundary conditions. In this case the conditions (3.13) and (3.14) guarantee that quadratic equation (3.16) has two distinct nonzero roots $z_{1}$ and $z_{2}$, so the matrix $A_{23}$ has two distinct eigenvalues $-z_{1},-z_{2}$. Let us fix a pair of corresponding eigenvectors $\left(\begin{array}{l}\alpha_{1} \\ \alpha_{2}\end{array}\right)$ and $\left(\begin{array}{l}\beta_{1} \\ \beta_{2}\end{array}\right)$. Then

$$
\left(\begin{array}{l}
\alpha_{1} \\
\alpha_{2}
\end{array}\right) \text { and }\left(\begin{array}{l}
\beta_{1} \\
\beta_{2}
\end{array}\right) \text { are linearly independent }
$$

because $z_{1} \neq z_{2}$. Therefore, the matrix $\left(\begin{array}{ll}\alpha_{1} & \beta_{1} \\ \alpha_{2} & \beta_{2}\end{array}\right)$ is invertible; we set

$$
\left(\begin{array}{ll}
\alpha_{1}^{\prime} & \alpha_{2}^{\prime} \\
\beta_{1}^{\prime} & \beta_{2}^{\prime}
\end{array}\right):=\left(\begin{array}{ll}
\alpha_{1} & \beta_{1} \\
\alpha_{2} & \beta_{2}
\end{array}\right)^{-1}
$$

Moreover, in view of Lemma 4 , the vectors $\left(\begin{array}{c}\alpha_{1} z_{1} \\ \alpha_{2}\end{array}\right)$ and $\left(\begin{array}{c}\beta_{1} z_{2} \\ \beta_{2}\end{array}\right)$ are solutions of the system (3.15).

Let $\tau_{1}$ and $\tau_{2}$ be chosen so that

$$
z_{1}=e^{i \pi \tau_{1}}, \quad z_{2}=e^{i \pi \tau_{2}}
$$

and

$$
\left|\operatorname{Re} \tau_{1}-\operatorname{Re} \tau_{2}\right| \leq 1, \quad\left|\operatorname{Re} \tau_{1}\right| \leq 1
$$


Then we have

$$
z_{1}=e^{i \pi \lambda} \quad \Leftrightarrow \quad \lambda=\tau_{1}+k, k \in 2 \mathbb{Z}
$$

and

$$
z_{2}=e^{i \pi \lambda} \quad \Leftrightarrow \quad \lambda=\tau_{2}+m, m \in 2 \mathbb{Z} .
$$

The right-hand sides of (3.22) and (3.23) give all eigenvalues of $L^{0}$. For each $\lambda$ in the two infinite series given by (3.22) and (3.23) we have an eigenvector of $L^{0}$ of the form (3.5) with

$$
\left(\begin{array}{l}
\xi \\
\eta
\end{array}\right)=\left(\begin{array}{c}
\alpha_{1} z_{1} \\
\alpha_{2}
\end{array}\right) \quad \text { if } \lambda=\tau_{1}+m, \quad\left(\begin{array}{l}
\xi \\
\eta
\end{array}\right)=\left(\begin{array}{c}
\beta_{1} z_{2} \\
\beta_{2}
\end{array}\right) \quad \text { if } \lambda=\tau_{2}+m \text {. }
$$

Thus, the operator $L^{0}$ subject to the boundary conditions (3.4) with matrix (3.11), has the following two series of eigenvectors:

$$
\Phi^{1}=\left\{\varphi_{k}^{1}, k \in 2 \mathbb{Z}\right\}, \quad \varphi_{k}^{1}:=\left(\begin{array}{c}
z_{1} \alpha_{1} e^{-i\left(\tau_{1}+k\right) x} \\
\alpha_{2} e^{i\left(\tau_{1}+k\right) x}
\end{array}\right)=\left(\begin{array}{c}
\alpha_{1} e^{i \tau_{1}(\pi-x)} e^{-i k x} \\
\alpha_{2} e^{i \tau_{1} x} e^{i k x}
\end{array}\right)
$$

and

$$
\Phi^{2}=\left\{\varphi_{m}^{2}, m \in 2 \mathbb{Z}\right\}, \quad \varphi_{m}^{2}:=\left(\begin{array}{c}
z_{2} \beta_{1} e^{-i\left(\tau_{2}+m\right) x} \\
\beta_{2} e^{i\left(\tau_{2}+m\right) x}
\end{array}\right)=\left(\begin{array}{c}
\beta_{1} e^{i \tau_{2}(\pi-x)} e^{-i m x} \\
\beta_{2} e^{i \tau_{2} x} e^{i m x}
\end{array}\right)
$$

Lemma 5. The system $\Phi=\Phi^{1} \cup \Phi^{2}$ is a Riesz basis in the space $L^{2}\left(I, \mathbb{C}^{2}\right), I=[0, \pi]$. Its biorthogonal system is $\tilde{\Phi}=\tilde{\Phi}^{1} \cup \tilde{\Phi}^{2}$, where

$$
\tilde{\Phi}^{1}=\left\{\tilde{\varphi}_{k}^{1}, k \in 2 \mathbb{Z}\right\}, \quad \tilde{\varphi}_{k}^{1}:=\left(\begin{array}{c}
\overline{\alpha_{1}^{\prime}} e^{i \overline{\tau_{1}}(\pi-x)} e^{-i k x} \\
\overline{\alpha_{2}^{\prime}} e^{i \overline{\tau_{1}} x} e^{i k x}
\end{array}\right),
$$

and

$$
\tilde{\Phi}^{2}=\left\{\tilde{\varphi}_{m}^{2}, m \in 2 \mathbb{Z}\right\}, \quad \tilde{\varphi}_{m}^{2}:=\left(\begin{array}{c}
\overline{\beta_{1}^{\prime}} e^{i \overline{\tau_{2}}(\pi-x)} e^{-i m x} \overline{\beta_{2}^{\prime}} e^{i \overline{\tau_{2}} x} e^{i m x}
\end{array}\right),
$$

with $\alpha_{1}^{\prime}, \alpha_{2}^{\prime}, \beta_{1}^{\prime}, \beta_{2}^{\prime}$ coming from (3.19).

Proof. The system $E=E^{1} \cup E^{2}$, where

$$
E^{\nu}=\left\{e_{k}^{\nu}, k \in 2 \mathbb{Z}\right\} ; \quad \nu=1,2 ; \quad e_{k}^{1}:=\left(\begin{array}{c}
e^{i k x} \\
0
\end{array}\right), \quad e_{m}^{2}:=\left(\begin{array}{c}
0 \\
e^{i m x}
\end{array}\right),
$$

is an orthonormal basis in $L^{2}\left(I, \mathbb{C}^{2}\right)$.

Consider the operator $A: L^{2}\left(I, \mathbb{C}^{2}\right) \rightarrow L^{2}\left(I, \mathbb{C}^{2}\right)$ defined by

$$
A\left(\begin{array}{l}
f \\
g
\end{array}\right)=\left(\begin{array}{c}
\alpha_{1} e^{i \tau_{1}(\pi-x)} f(\pi-x) \\
\alpha_{2} e^{i \tau_{1} x} f(x)
\end{array}\right)+\left(\begin{array}{c}
\beta_{1} e^{i \tau_{2}(\pi-x)} g(\pi-x) \\
\beta_{2} e^{i \tau_{2} x} g(x)
\end{array}\right) .
$$


Since we have $\Phi=A(E)$, the lemma will be proved if we show that $A$ is an isomorphism. Since the functions $e^{i \tau_{\nu} x}$ and $e^{i \tau_{\nu}(\pi-x)}, \nu=1,2$, are bounded, it follows that $A$ is bounded operator. Let us find its inverse. By (3.29), the equation

$$
A\left(\begin{array}{l}
f \\
g
\end{array}\right)=\left(\begin{array}{l}
F \\
G
\end{array}\right)
$$

is equivalent to the following system of two linear equations in two unknowns $f, g$ :

$$
\begin{array}{r}
\alpha_{1} e^{i \tau_{1} x} f(x)+\beta_{1} e^{i \tau_{2} x} g(x)=F(\pi-x), \\
\alpha_{2} e^{i \tau_{1} x} f(x)+\beta_{2} e^{i \tau_{2} x} g(x)=G(x) .
\end{array}
$$

By (3.18) and (3.19), we get

$$
\left(\begin{array}{c}
e^{i \tau_{1} x} f(x) \\
e^{i \tau_{2} x} g(x)
\end{array}\right)=\left(\begin{array}{c}
\alpha_{1}^{\prime} F(\pi-x)+\alpha_{2}^{\prime} G(x) \\
\beta_{1}^{\prime} F(\pi-x)+\beta_{2}^{\prime} G(x)
\end{array}\right),
$$

which leads to

$$
A^{-1}\left(\begin{array}{l}
F \\
G
\end{array}\right)=\left(\begin{array}{l}
e^{-i \tau_{1} x}\left[\alpha_{1}^{\prime} F(\pi-x)+\alpha_{2}^{\prime} G(x)\right] \\
e^{-i \tau_{2} x}\left[\beta_{1}^{\prime} F(\pi-x)+\beta_{2}^{\prime} G(x)\right]
\end{array}\right),
$$

Now it is easy to see that $A^{-1}$ is bounded.

Let us find the adjoint operator of $A^{-1}$. Since

$$
\begin{gathered}
\left\langle A^{-1}\left(\begin{array}{l}
F \\
G
\end{array}\right),\left(\begin{array}{l}
f \\
0
\end{array}\right)\right\rangle=\frac{1}{\pi} \int_{0}^{\pi}\left\{\left[\alpha_{1}^{\prime} F(\pi-x)+\alpha_{2}^{\prime} G(x)\right] e^{-i \tau_{1} x} \overline{f(x)} d x\right. \\
=\frac{1}{\pi} \int_{0}^{\pi}\left(F(x) \overline{\overline{\alpha_{1}^{\prime}}} f(\pi-x) e^{i \overline{\tau_{1}}(\pi-x)}+G(x) \overline{\overline{\alpha_{2}^{\prime}}} f(x) e^{i \overline{\bar{\tau}_{1}} x}\right) d x
\end{gathered}
$$

we get

$$
\left(A^{-1}\right)^{*}\left(\begin{array}{l}
f \\
0
\end{array}\right)=\left(\begin{array}{c}
\overline{\alpha_{1}^{\prime}} f(\pi-x) e^{i \overline{\tau_{1}}(\pi-x)} \overline{\alpha_{2}^{\prime}} f(x) e^{i \overline{\tau_{1}} x}
\end{array}\right) .
$$

In an analogous way it follows that

$$
\left(A^{-1}\right)^{*}\left(\begin{array}{l}
0 \\
g
\end{array}\right)=\left(\begin{array}{c}
\overline{\beta_{1}^{\prime}} g(\pi-x) e^{i \overline{\tau_{2}}(\pi-x)} \overline{\beta_{2}^{\prime}} g(x) e^{i \overline{\tau_{2}} x}
\end{array}\right)
$$

Thus,

$$
\left(A^{-1}\right)^{*}\left(\begin{array}{l}
f \\
g
\end{array}\right)=\left(\begin{array}{c}
\overline{\alpha_{1}^{\prime}} f(\pi-x) e^{i \overline{\tau_{1}}(\pi-x)} \\
\overline{\alpha_{2}^{\prime}} f(x) e^{i \overline{\tau_{1}} x}
\end{array}\right)+\left(\begin{array}{c}
\overline{\beta_{1}^{\prime}} g(\pi-x) e^{i \overline{\tau_{2}}(\pi-x)} \\
\overline{\beta_{2}^{\prime}} g(x) e^{i \overline{\tau_{2}} x}
\end{array}\right) .
$$

By (2.3), the system $\Phi$ is a Riesz basis, and its biorthogonal system is given by (3.26) and (3.27). This completes the proof. 
The system $\tilde{\Phi}$ has the same form as $\Phi$, so it is a system of eigenvectors of $L^{0}$ subject to appropriate boundary conditions. Indeed, let $S$ denote the matrix $\left(\begin{array}{ll}b & a \\ d & c\end{array}\right)$, and let

$$
\left(S^{-1}\right)^{*}=\left(\begin{array}{cc}
\tilde{b} & \tilde{a} \\
\tilde{d} & \tilde{c}
\end{array}\right)
$$

If $\left(\begin{array}{l}\alpha_{1} \\ \alpha_{2}\end{array}\right)$ and $\left(\begin{array}{l}\beta_{1} \\ \beta_{2}\end{array}\right)$ are eigenvectors of $S$ corresponding to $-z_{1}$ and $-z_{2}$ as in (3.18) and Lemma 4, then

$$
S\left(\begin{array}{ll}
\alpha_{1} & \beta_{1} \\
\alpha_{2} & \beta_{2}
\end{array}\right)=\left(\begin{array}{ll}
-z_{1} \alpha_{1} & -z_{2} \beta_{1} \\
-z_{1} \alpha_{2} & -z_{2} \beta_{2}
\end{array}\right) .
$$

Let us mention that the relation (3.34) determines the matrix $S$ if numbers $z_{1} \neq z_{2}$ and $\alpha_{1}, \alpha_{2}, \beta_{1}, \beta_{2}$ satisfying (3.18) are given.

In view of (3.24), taking the inverse matrices of both sides of (3.34), and then passing to adjoint matrices, we get

$$
\left(S^{-1}\right)^{*}\left(\begin{array}{ll}
\overline{\alpha_{1}^{\prime}} & \overline{\beta_{1}^{\prime}} \\
\overline{\alpha_{2}^{\prime}} & \overline{\beta_{2}^{\prime}}
\end{array}\right)=\left(\begin{array}{ll}
-\frac{1}{\overline{z_{1}}} \overline{\alpha_{1}^{\prime}} & -\frac{1}{\bar{z}_{2}} \overline{\beta_{1}^{\prime}} \\
-\frac{1}{\overline{z_{1}}} \overline{\alpha_{2}^{\prime}} & -\frac{1}{\overline{z_{2}}} \overline{\beta_{2}^{\prime}}
\end{array}\right) .
$$

This means that $-1 / \overline{z_{1}},-1 / \overline{z_{2}}$ are the eigenvalues of the matrix $\left(S^{-1}\right)^{*}$, and $\left(\frac{\overline{\alpha_{1}^{\prime}}}{\overline{\alpha_{2}^{\prime}}}\right),\left(\frac{\overline{\beta_{1}^{\prime}}}{\overline{\beta_{2}^{\prime}}}\right)$ is a pair of corresponding (linearly independent) eigenvectors.

Consider the boundary conditions that correspond to the matrix

$$
\left(\begin{array}{cccc}
1 & \tilde{b} & \tilde{a} & 0 \\
0 & \tilde{d} & \tilde{c} & 1
\end{array}\right)
$$

with $\tilde{b}, \tilde{a}, \tilde{d}, \tilde{c}$ coming from (3.33). In view of Lemma 4, $1 / \overline{z_{1}}$ and $1 / \overline{z_{2}}$ are the roots of the characteristic equation (3.15). But if $z=e^{i \tau \pi}$ then $1 / \bar{z}=\overline{e^{-i \tau \pi}}=e^{i \bar{\tau} \pi}$. Now, by (3.24) $-(3.27)$, it follows that $\tilde{\Phi}$ is a system of eigenvectors of $L^{0}$ subject to the boundary conditions (3.35).

Next we show that, as usual, the biorthogonal system $\tilde{\Phi}$ is the system of eigenvectors of the adjoint operator $\left(L_{b c}^{0}\right)^{*}$ (or, which is the same, of $L^{0}$ subject to adjoint boundary conditions $\left.b c^{*}\right)$.

Lemma 6. Let $L_{b c}^{0}$ be a closed operator with boundary conditions bc defined by (3.10) and (3.11). Then its adjoint operator $\left(L_{b c}\right)^{*}$ is $L_{b c^{*}}^{0}$, where the boundary conditions bc* are given by the matrix (3.35). 
Proof. With $f=\left(\begin{array}{l}f_{1} \\ f_{2}\end{array}\right)$ and $g=\left(\begin{array}{l}g_{1} \\ g_{2}\end{array}\right)$ such that $f, g \in H^{1}\left(I, \mathbb{C}^{2}\right)$ we have

$$
\begin{gathered}
\left\langle L^{0} f, g\right\rangle-\left\langle f, L^{0} g\right\rangle=\frac{i}{\pi} \int_{0}^{\pi} \frac{d}{d x}\left(f_{1}(x) \overline{g_{1}(x)}-f_{2}(x) \overline{g_{2}(x)}\right) d x \\
=\frac{i}{\pi}\left(f_{1}(\pi) \overline{g_{1}(\pi)}-f_{1}(0) \overline{g_{1}(0)}-f_{2}(\pi) \overline{g_{2}(\pi)}+f_{2}(0) \overline{g_{2}(0)}\right) \\
=\frac{i}{\pi}\left(f_{1}(\pi) \overline{g_{1}(\pi)}+\left(b f_{1}(\pi)+a f_{2}(0)\right) \overline{g_{1}(0)}+\left(d f_{1}(\pi)+c f_{2}(0)\right) \overline{g_{2}(\pi)}+f_{2}(0) \overline{g_{2}(0)}\right) \\
=\frac{i}{\pi}\left(f_{1}(\pi) \overline{\left[\bar{b} g_{1}(0)+g_{1}(\pi)+\bar{d} g_{2}(\pi)\right]}+f_{2}(0) \overline{\left[\bar{a} g_{1}(0)+g_{2}(0)+\bar{c} g_{2}(\pi)\right]}\right) .
\end{gathered}
$$

In view of (3.10), one can easily see that $f_{1}(\pi)$ and $f_{2}(0)$ could be any numbers.

Therefore, the boundary conditions of the adjoint operator are determined by the matrix

$$
\left(\begin{array}{cccc}
\bar{b} & 1 & 0 & \bar{d} \\
\bar{a} & 0 & 1 & \bar{c}
\end{array}\right)
$$

In view of (3.33), if we bring it to the equivalent form (3.11) multiplying from the left by $\left(\begin{array}{ll}\bar{b} & \bar{d} \\ \bar{a} & \bar{c}\end{array}\right)^{-1}$, the result will be just (3.35) .

3. Dirichlet-type boundary conditions. In general, for strictly regular $b c$, the spectrum of the operator $L_{b c}^{0}$ consists of two arithmetic progressions (3.22) and (3.23), with difference $=2$. If

$$
b+c=0,
$$

then the equation (3.16) has the following two roots

$$
z_{1}=\sqrt{a d-b c}, \quad z_{2}=-z_{1} .
$$

In view of (3.20), in this case we have $z_{1}=e^{i \tau_{1} \pi}$ and $z_{2}=e^{i \tau_{2} \pi}$ with $\tau_{2}=\tau_{1} \pm 1$. Therefore, the union of the corresponding two arithmetic progressions (3.22) and (3.23) gives the spectrum of $L_{b c}^{0}$ in the form of one arithmetic progression with difference 1 :

$$
\lambda=\tau_{1}+m, \quad m \in \mathbb{Z} .
$$

We call boundary conditions with the property (3.37) Dirichlet-type boundary conditions.

For Dirichlet-type $b c$, the adjoint boundary conditions $b c^{*}$ are also Dirichlet-type. Indeed, in view of (3.16), $b c$ given by a matrix (3.11) are Dirichlet-type if and only if we have $z_{1}+z_{2}=0$, where $z_{1}$ and $z_{2}$ are the roots of (3.16). By Lemma 6 and the discussion after (3.35), 
the roots of the equation (3.16) that corresponds to $b c^{*}$ are $1 / \overline{z_{1}}, 1 / \overline{z_{2}}$, so we have

$$
\frac{1}{\overline{z_{1}}}+\frac{1}{\overline{z_{2}}}=\frac{\overline{z_{1}+z_{2}}}{\overline{z_{1} z_{2}}}=0 .
$$

Therefore, $b c^{*}$ are Dirichlet-type also.

4. Regular but not strictly regular boundary conditions.

Now we assume that (3.13) holds, but (3.14) fails, i.e.,

$$
(b+c)^{2}-4(b c-a d)=(b-c)^{2}+4 a d=0 .
$$

In this case the characteristic equation (3.16) has one double root:

$$
z_{*}=-(b+c) / 2 \text {. }
$$

Notice, that $z_{*} \neq 0$ because otherwise (3.40) would imply $b c-a d=0$ which contradicts to the regularity condition (3.13).

Let $\tau_{*}$ be chosen so that

$$
z_{*}=-(b+c) / 2=e^{i \pi \tau_{*}}, \quad\left|R e \tau_{*}\right| \leq 1 .
$$

Then all eigenvalues of $L_{b c}^{0}$ are given by

$$
\operatorname{Sp}\left(L_{b c}^{0}\right)=\left\{\tau_{*}+k, \quad k \in 2 \mathbb{Z}\right\} .
$$

In view of Lemma 4, the corresponding eigenvectors have the form (3.5) with $\left(\begin{array}{l}\xi \\ \eta\end{array}\right)=\left(\begin{array}{c}\alpha_{1} z_{*} \\ \alpha_{2}\end{array}\right)$, where $\left(\begin{array}{l}\alpha_{1} \\ \alpha_{2}\end{array}\right)$ is an eigenvector of the matrix $A_{23}=\left[\begin{array}{ll}b & a \\ d & c\end{array}\right]$ corresponding to its double eigenvalue $-z_{*}$, i.e.,

$$
\left(A_{23}+z_{*} I\right)\left(\begin{array}{l}
\alpha_{1} \\
\alpha_{2}
\end{array}\right)=\left[\begin{array}{cc}
b+z_{*} & a \\
d & c+z_{*}
\end{array}\right]\left(\begin{array}{l}
\alpha_{1} \\
\alpha_{2}
\end{array}\right)=\left[\begin{array}{cc}
\frac{b-c}{2} & a \\
d & \frac{c-b}{2}
\end{array}\right]\left(\begin{array}{l}
\alpha_{1} \\
\alpha_{2}
\end{array}\right)=0 .
$$

The matrix $A_{23}$ will have two linearly independent eigenvectors $\left(\begin{array}{l}\alpha_{1} \\ \alpha_{2}\end{array}\right)$ and $\left(\begin{array}{c}\beta_{1} \\ \beta_{2}\end{array}\right)$ if and only if $A_{23}+z_{*} I$ is the zero matrix, i.e.,

$$
b=c, \quad a=0, \quad d=0 .
$$

Then the matrix (3.11) has the form

$$
\left[\begin{array}{cccc}
1 & b & 0 & 0 \\
0 & 0 & b & 1
\end{array}\right], \quad b \neq 0 .
$$

We call the boundary conditions given by the matrix (3.11) periodictype if (3.45) holds, i.e., bc is defined by (3.46). Using the same argument as in the strictly regular case we get the following lemma. 
Lemma 7. For periodic-type bc, (3.22) with $\tau_{1}=\tau_{*}$ gives all eigenvalues of $L_{b c}^{0}$, and each eigenvalue is of geometric multiplicity 2. There are linearly independent vectors $\left(\begin{array}{l}\alpha_{1} \\ \alpha_{2}\end{array}\right)$ and $\left(\begin{array}{l}\beta_{1} \\ \beta_{2}\end{array}\right)$ such that the system $\Phi=\Phi^{1} \cup \Phi^{2}$, given by (3.24) and (3.25) with $\tau_{2}=\tau_{1}=\tau_{*}$, is a Riesz basis in the space $L^{2}\left(I, \mathbb{C}^{2}\right), I=[0, \pi]$. Its biorthogonal system $\tilde{\Phi}=\tilde{\Phi}^{1} \cup \tilde{\Phi}^{2}$ is defined by (3.26) and (3.27).

Next we consider the case when (3.40) holds but (3.45) fails, i.e.,

$$
|b-c|+|a|+|d|>0 \text {. }
$$

As we will see below, in this case each eigenvalue of $L_{b c}^{0}$ is of algebraic multiplicity 2 but of geometric multiplicity 1, i.e., associated eigenvectors appear. Here we have the following subcases:

(i) If $a=0$, then (3.40) implies $b=c$, and by (3.47) we have $d \neq 0$. By the regularity condition (3.13) we have $b c-a d \neq 0$, which yields $b \neq 0$. In other words, the matrix (3.11) has the form

$$
\left[\begin{array}{llll}
1 & b & 0 & 0 \\
0 & d & b & 1
\end{array}\right], \quad d, b \neq 0 .
$$

Here we choose the following solution of (3.44)

$$
\alpha_{1}=0, \quad \alpha_{2}=d \text {. }
$$

(ii) If $d=0$, then (3.40) implies $b=c$, and by (3.47) we have $a \neq 0$. Now the matrix (3.11) has the form

$$
\left[\begin{array}{llll}
1 & b & a & 0 \\
0 & 0 & b & 1
\end{array}\right], \quad d, b \neq 0 .
$$

Here we choose the following solution of (3.44):

$$
\alpha_{1}=a, \quad \alpha_{2}=0 .
$$

(iii) If $a, d \neq 0$, then (3.40) implies $b \neq c$. Here we choose the following solution of (3.44):

$$
\alpha_{1}=a, \quad \alpha_{2}=(c-b) / 2 .
$$

Of course, (3.43) gives all eigenvalues. A corresponding system of eigenvectors is

$$
\varphi_{k}^{1}=\left(\begin{array}{c}
\alpha_{1} e^{i \tau_{*}(\pi-x)} e^{-i k x} \\
\alpha_{2} e^{i \tau_{*} x} e^{i k x}
\end{array}\right), \quad k \in 2 \mathbb{Z},
$$

where $\alpha_{1}, \alpha_{2}$ are given, respectively, by (3.49), (3.51) and (3.52).

We look for a system of associated eigenvectors of the form

$$
\varphi_{k}^{2}=\left(\begin{array}{c}
\left(\beta_{1}-\alpha_{1} x\right) e^{i \tau_{*}(\pi-x)} e^{-i k x} \\
\left(\beta_{2}+\alpha_{2} x\right) e^{i \tau_{*} x} e^{i k x}
\end{array}\right), \quad k \in 2 \mathbb{Z} .
$$


Then $L^{0} \varphi_{k}^{2}=\lambda_{k} \varphi_{k}^{2}-i \varphi_{k}^{1}$, so $\varphi_{k}^{2}$ is an associated eigenvector if and only if it satisfies the boundary conditions. This leads to the following system of two linear equations in two unknowns $\beta_{1}$ and $\beta_{2}$ :

$$
\begin{aligned}
& \beta_{1} z_{*}+b\left(\beta_{1}-\pi \alpha_{1}\right)+a \beta_{2}=0 \\
& d\left(\beta_{1}-\pi \alpha_{1}\right)+c \beta_{2}+\left(\beta_{2}+\pi \alpha_{2}\right) z_{*}=0,
\end{aligned}
$$

or, equivalently,

$$
\begin{aligned}
& \left(z_{*}+b\right) \beta_{1}+a \beta_{2}=\pi b \alpha_{1} \\
& d \beta_{1}+\left(c+z_{*}\right) \beta_{2}=\pi d \alpha_{1}-\pi \alpha_{2} z_{*}
\end{aligned}
$$

By (3.41), $b+z_{*}=(b-c) / 2, c+z_{*}=(c-b) / 2$. Moreover, by (3.41) and (3.44),$d \alpha_{1}=\alpha_{2}(b-c) / 2$, and therefore, $\pi d \alpha_{1}-\pi \alpha_{2} z_{*}=\pi \alpha_{2} \frac{b-c}{2}+$ $\pi \alpha_{2} \frac{b+c}{2}=\pi b \alpha_{2}$.

Thus, (3.54) is a system of associated vectors if and only if $\beta_{1}$ and $\beta_{2}$ satisfy

$$
\left[\begin{array}{cc}
b+z_{*} & a \\
d & c+z_{*}
\end{array}\right]\left(\begin{array}{l}
\beta_{1} \\
\beta_{2}
\end{array}\right)=\left[\begin{array}{cc}
\frac{b-c}{2} & a \\
d & \frac{c-b}{2}
\end{array}\right]\left(\begin{array}{l}
\beta_{1} \\
\beta_{2}
\end{array}\right)=\left(\begin{array}{l}
\pi b \alpha_{1} \\
\pi b \alpha_{2}
\end{array}\right) .
$$

Notice, that (3.44) and (3.55) mean that $\left(\begin{array}{l}\alpha_{1} \\ \alpha_{2}\end{array}\right)$ is an eigenvalue of the matrix $A_{23}=\left[\begin{array}{ll}b & a \\ d & c\end{array}\right]$ corresponding to its double eigenvalue $-z_{*}$, and $\left(\begin{array}{l}\beta_{1} \\ \beta_{2}\end{array}\right)$ is an associated vector.

With $\alpha_{1}$ and $\alpha_{2}$ fixed, respectively, in (3.49), (3.51) and (3.52), we choose corresponding solutions of (3.55):

$$
\beta_{1}=\pi b, \quad \beta_{2}=0 \quad \text { in the case (i); }
$$

and

$$
\beta_{1}=0, \quad \beta_{2}=\pi b \quad \text { in the cases (ii) and (iii). }
$$

Lemma 8. The system $\Phi$ of eigenfunctions $\varphi_{k}^{1}, k \in 2 \mathbb{Z}$, and associated functions $\varphi_{k}^{2}, k \in 2 \mathbb{Z}$, given in (3.53) and (3.54), is a Riesz basis in the space $L^{2}\left(I, \mathbb{C}^{2}\right), I=[0, \pi]$. Its biorthogonal system is $\tilde{\Phi}=\left\{\tilde{\varphi}_{k}^{1}, \tilde{\varphi}_{k}^{2}, k \in\right.$ $2 \mathbb{Z}\}$, where

$\tilde{\varphi}_{k}^{1}=\left(\begin{array}{c}\bar{\Delta}^{-1} \overline{\alpha_{2}} e^{i \overline{\tau_{*}}(\pi-x)} e^{-i k x} \\ \bar{\Delta}^{-1} \overline{\alpha_{1}} e^{i \overline{\tau_{*}} x} e^{i k x}\end{array}\right), \quad \tilde{\varphi}_{k}^{2}=\left(\begin{array}{c}\bar{\Delta}^{-1}\left[\overline{\beta_{2}}+\overline{\alpha_{2}}(\pi-x)\right] e^{i \overline{\tau_{*}}(\pi-x)} e^{-i k x} \\ \bar{\Delta}^{-1}\left[\overline{\beta_{1}}-\overline{\alpha_{1}}(\pi-x)\right] e^{i \overline{\tau_{*}} x} e^{i k x}\end{array}\right)$

with $\Delta=\alpha_{1} \beta_{2}-\alpha_{2} \beta_{1}+\pi \alpha_{1} \alpha_{2}$. 
Proof. Consider the operator $A: L^{2}\left(I, \mathbb{C}^{2}\right) \rightarrow L^{2}\left(I, \mathbb{C}^{2}\right)$ defined by (3.59)

$$
A\left(\begin{array}{l}
f \\
g
\end{array}\right)=\left(\begin{array}{c}
\alpha_{1} e^{i \tau_{*}(\pi-x)} f(\pi-x) \\
\alpha_{2} e^{i \tau_{*} x} f(x)
\end{array}\right)+\left(\begin{array}{c}
\left(\beta_{1}-\alpha_{1} x\right) e^{i \tau_{*}(\pi-x)} g(\pi-x) \\
\left(\beta_{2}+\alpha_{2} x\right) e^{i \tau_{*} x} g(x)
\end{array}\right) .
$$

Since we have $\Phi=A(E)$, where $E$ is the orthonormal basis (3.28), the lemma will be proved if we show that $A$ is an isomorphism. One can easily see that $A$ is bounded operator. Let us find its inverse. By (3.59), the equation

$$
A\left(\begin{array}{l}
f \\
g
\end{array}\right)=\left(\begin{array}{l}
F \\
G
\end{array}\right)
$$

is equivalent to the following system of two linear equations in two unknowns $f, g$ :

$$
\begin{aligned}
\alpha_{1} f(x)+ & \left(\beta_{1}-\alpha_{1}[\pi-x]\right) g(x)=F(\pi-x) e^{-i \tau_{*} x}, \\
& \alpha_{2} f(x)+\left(\beta_{2}+\alpha_{2} x\right) g(x)=G(x) e^{-i \tau_{*} x} .
\end{aligned}
$$

The determinant of this system is

$$
\Delta=\operatorname{det}\left[\begin{array}{cc}
\alpha_{1} & \beta_{1}-\pi \alpha_{1}+\alpha_{1} x \\
\alpha_{2} & \beta_{2}+\alpha_{2} x
\end{array}\right]= \begin{cases}-\pi b d & \text { in case (i) } \\
\pi a b & \text { in case (ii) } \\
\pi a(b+c) / 2 & \text { in case (iii) }\end{cases}
$$

due to our choices of $\alpha_{1}, \alpha_{2}$ in (3.49), (3.51), (3.52) and $\beta_{1}, \beta_{2}$ in (3.56) and (3.57). Thus we get

$$
\Delta\left(\begin{array}{c}
f(x) \\
g(x)
\end{array}\right)=\left(\begin{array}{c}
{\left[\left(\beta_{2}+\alpha_{2} x\right) F(\pi-x)-\left(\beta_{1}-\pi \alpha_{1}+\alpha_{1} x\right) G(x)\right] e^{-i \tau_{*} x}} \\
{\left[-\alpha_{2} F(\pi-x)+\alpha_{1} G(x)\right] e^{-i \tau_{*} x}}
\end{array}\right)
$$

which implies (since $\Delta \neq 0$ )

$$
A^{-1}\left(\begin{array}{c}
F \\
G
\end{array}\right)=\frac{1}{\Delta}\left(\begin{array}{c}
{\left[\left(\beta_{2}+\alpha_{2} x\right) F(\pi-x)-\left(\beta_{1}-\pi \alpha_{1}+\alpha_{1} x\right) G(x)\right] e^{-i \tau_{*} x}} \\
{\left[-\alpha_{2} F(\pi-x)+\alpha_{1} G(x)\right] e^{-i \tau_{*} x}}
\end{array}\right) .
$$

Now it is easy to see that the operator $A^{-1}$ is bounded.

A simple calculation (similar to the one used in Lemma 6) shows that the adjoint operator of $A^{-1}$ is

$$
\left(A^{-1}\right)^{*}\left(\begin{array}{l}
f \\
g
\end{array}\right)=\bar{\Delta}^{-1}\left(\begin{array}{c}
{\left[\left(\overline{\beta_{2}}+\overline{\alpha_{2}} \pi-\overline{\alpha_{2}} x\right) f(\pi-x)+\overline{\alpha_{2}} g(\pi-x)\right] e^{i \overline{\tau_{*}}(\pi-x)}} \\
\left.\left[-\overline{\beta_{1}}-\overline{\alpha_{1}} \pi+\overline{\alpha_{1}} x\right) f(x)+\overline{\alpha_{1}} g(x)\right] e^{i \overline{\tau_{*}} x}
\end{array}\right) .
$$

Since we have $\tilde{\Phi}=\left(A^{-1}\right)^{*}(E)$, where $E$ is the orthonormal basis defined in (3.28), the family $\tilde{\Phi}$ is the biorthogonal system to $\Phi$. 


\section{Matrix Representation of $L_{b c}$ And its Resolvent $R_{b c}(\lambda)$}

Next we consider, for arbitrary regular $b c$, the Fourier representation of $L_{b c}$ and its resolvent $L_{b c}(\lambda)$ with respect to a corresponding Riesz basis consisting of eigenfunctions and associated functions of the operator $L_{b c}^{0}$ (constructed in Lemmas 5, 7, 8).

1. Let $V: L^{2}\left(I, \mathbb{C}^{2}\right) \rightarrow L^{2}\left(I, \mathbb{C}^{2}\right)$ be the operator of multiplication by the matrix $v(x)=\left[\begin{array}{cc}0 & P(x) \\ Q(x) & 0\end{array}\right]$, i.e.,

$$
V\left(\begin{array}{l}
y_{1} \\
y_{2}
\end{array}\right)=\left[\begin{array}{cc}
0 & P(x) \\
Q(x) & 0
\end{array}\right]\left(\begin{array}{l}
y_{1} \\
y_{2}
\end{array}\right)=\left(\begin{array}{c}
P y_{2} \\
Q y_{1}
\end{array}\right) \text {. }
$$

For a regular boundary condition $b c$, let $\Phi=\left\{\varphi_{k}^{1}, \varphi_{k}^{2}, k \in \mathbb{Z}\right\}$ and $\tilde{\Phi}=\left\{\tilde{\varphi}_{k}^{1}, \tilde{\varphi}_{k}^{2}, k \in \mathbb{Z}\right\}$ be the corresponding Riesz basis (consisting of eigenfunctions and associated functions of the operator $L_{b c}^{0}$ ) and its biorthogonal system constructed, respectively, in Lemma 5 if $b c$ is strictly regular, in Lemma 7 if $b c$ is periodic type, and in Lemma 8 otherwise. In this section and thereafter, we consider matrix representation with respect to that basis only.

Lemma 9. The matrix representation of $V$ with respect to the basis $\Phi$ has the form

$$
\begin{gathered}
V \sim\left[\begin{array}{ll}
V^{11} & V^{12} \\
V^{21} & V^{22}
\end{array}\right], \quad V^{\mu \nu}=\left(V_{j k}^{\mu \nu}\right)_{j, k \in 2 \mathbb{Z}}, \quad \mu, \nu \in\{1,2\}, \\
V_{j k}^{\mu \nu}=\left\langle V \varphi_{k}^{\nu}, \tilde{\varphi}_{j}^{\mu}\right\rangle=w^{\mu \nu}(j+k),
\end{gathered}
$$

where

$$
w^{\mu \nu}=\left(w^{\mu \nu}(m)\right) \in \ell^{2}(2 \mathbb{Z}), \quad\left\|w^{\mu \nu}\right\|_{\ell^{2}} \leq C\left(\|P\|_{L^{2}}+\|Q\|_{L^{2}}\right),
$$

with $C=C(\Phi, \tilde{\Phi})$.

Proof. We consider only the case where $\mu=1, \nu=2$ because the proof is similar in the other three cases.

If $b c$ is strictly regular, then we get, by (3.24)-(3.27),

$$
\begin{aligned}
& V_{j k}^{12}=\left\langle V \varphi_{k}^{2}, \tilde{\varphi}_{j}^{1}\right\rangle=\left\langle\left(\begin{array}{c}
P(x) \beta_{2} e^{i \tau_{2} x} e^{i k x} \\
Q(x) \beta_{1} e^{i \tau_{2}(\pi-x)} e^{-i k x}
\end{array}\right),\left(\begin{array}{c}
\overline{\alpha_{1}^{\prime}} e^{i \overline{\tau_{1}}(\pi-x)} e^{-i j x} \\
\overline{\alpha_{2}^{\prime}} e^{i \overline{\tau_{1}} x} e^{i j x}
\end{array}\right)\right\rangle \\
= & \frac{1}{\pi} \int_{0}^{\pi}\left[\alpha_{1}^{\prime} \beta_{2} e^{i\left(\tau_{2} x+\tau_{1}(x-\pi)\right)} P(x) e^{i(j+k) x} d x+\alpha_{2}^{\prime} \beta_{1} e^{i\left(\tau_{2}(\pi-x)-\tau_{1} x\right)} Q(x) e^{-i(j+k) x}\right] d x .
\end{aligned}
$$

Therefore, (4.2) holds for $\mu=1, \nu=2$ with

$$
w^{12}(m):=p^{12}(-m)+q^{12}(m), \quad m \in 2 \mathbb{Z},
$$


where $p^{12}(m)$ and $q^{12}(m), m \in 2 \mathbb{Z}$, are the Fourier coefficients of the functions $g^{12}(x) P(x)$ and $h^{12}(x) Q(x)$, with

$$
g^{12}(x):=\alpha_{1}^{\prime} \beta_{2} e^{i\left(\tau_{2} x+\tau_{1}(x-\pi)\right)}, \quad h^{12}(x):=\alpha_{2}^{\prime} \beta_{1} e^{i\left(\tau_{2}(\pi-x)-\tau_{1} x\right)} .
$$

By the Parseval identity,

$$
\sum_{m}\left|p^{12}(m)\right|^{2}=\left\|g^{12}(x) P(x)\right\|_{L^{2}(I)}^{2} \leq \sup _{[0, \pi]}\left|g^{12}(x)\right|^{2} \cdot\|P\|_{L^{2}(I)}^{2}
$$

and

$$
\sum_{m}\left|q^{12}(m)\right|^{2}=\left\|h^{12}(x) Q(x)\right\|_{L^{2}(I)}^{2} \leq \sup _{[0, \pi]}\left|h^{12}(x)\right|^{2} \cdot\|Q\|_{L^{2}(I)}^{2} .
$$

Thus, (4.3) holds with a constant $C$ depending on the parameters $\alpha_{1}^{\prime}, \alpha_{2}^{\prime}, \beta_{1}, \beta_{2}, \tau_{1}, \tau_{2}$.

The proof is exactly the same if $b c$ is periodic type (the same formulas work but with $\tau_{2}=\tau_{1}=\tau_{*}$ ).

If $b c$ is not strictly regular and not of periodic type, then by (3.54) and (3.58) we have

$$
\begin{aligned}
& \left\langle V \varphi_{k}^{2}, \tilde{\varphi}_{k}^{1}\right\rangle=\left\langle\left(\begin{array}{c}
P(x)\left(\beta_{2}+\alpha_{2} x\right) e^{i \tau_{*} x} e^{i k x} \\
Q(x)\left(\beta_{1}-\alpha_{1} x\right) e^{i \tau_{*}(\pi-x)} e^{-i k x}
\end{array}\right),\left(\begin{array}{c}
\bar{\Delta}^{-1} \overline{\alpha_{2}} e^{i \overline{\tau_{*}}(\pi-x)} e^{-i j x} \\
\bar{\Delta}^{-1} \overline{\alpha_{1}} e^{i \overline{\tau_{*}} x} e^{i j x}
\end{array}\right)\right\rangle \\
& =\frac{1}{\pi} \int_{0}^{\pi}\left[P(x) \frac{\alpha_{2}}{\Delta}\left(\beta_{2}+\alpha_{2} x\right) e^{i \tau_{*}(2 x-\pi)} e^{i(j+k) x}\right] d x \\
& +\frac{1}{\pi} \int_{0}^{\pi}\left[Q(x) \frac{\alpha_{1}}{\Delta}\left(\beta_{1}-\alpha_{1} x\right) e^{i \tau_{*}(\pi-2 x)} e^{-i(j+k) x}\right] d x .
\end{aligned}
$$

Therefore, (4.2) holds for $\mu=1, \nu=2$ with

$$
w^{12}(m):=p_{1}^{12}(-m)+q_{1}^{12}(m), \quad m \in 2 \mathbb{Z},
$$

where $p_{1}^{12}(m)$ and $q_{1}^{12}(m), m \in 2 \mathbb{Z}$, are the Fourier coefficients of the functions $g_{1}^{12}(x) P(x)$ and $h_{1}^{12}(x) Q(x)$, with

$$
g_{1}^{12}(x):=\frac{\alpha_{2}}{\Delta}\left(\beta_{2}+\alpha_{2} x\right) e^{i \tau_{*}(2 x-\pi)}, \quad h_{1}^{12}(x):=\frac{\alpha_{1}}{\Delta}\left(\beta_{1}-\alpha_{1} x\right) e^{i \tau_{*}(\pi-2 x)} .
$$

Since these functions are bounded, again the Parseval identity implies (4.3) with a constant $C$ depending on parameters $\alpha_{1}, \alpha_{2}, \beta_{1}, \beta_{2}, \tau_{*}$.

2. If $b c$ is strictly regular boundary condition, then by (3.22) and (3.23) the spectrum of $L_{b c}^{0}$ consists of two disjoint sequences

$$
S p\left(L_{b c}^{0}\right)=\left\{\tau_{1}+k, k \in 2 \mathbb{Z}\right\} \cup\left\{\tau_{2}+k, k \in 2 \mathbb{Z}\right\} .
$$


The resolvent operator $R_{b c}^{0}(\lambda)=\left(\lambda-L_{b c}^{0}\right)^{-1}$ is well defined for $\lambda \notin$ $S p\left(L_{b c}^{0}\right)$, and we have

$$
R_{b c}^{0}(\lambda) \varphi_{k}^{\mu}=\frac{1}{\lambda-\tau_{\mu}-k} \varphi_{k}^{\mu}, \quad k \in 2 \mathbb{Z}, \quad \mu=1,2 .
$$

By (3.43), for regular but not strictly regular $b c$ the spectrum of $L_{b c}^{0}$ is given by

$$
\operatorname{Sp}\left(L_{b c}^{0}\right)=\left\{\tau_{*}+k, \quad k \in 2 \mathbb{Z}\right\},
$$

where each eigenvalue is of algebraic multiplicity 2 . The resolvent operator $R_{b c}^{0}(\lambda)=\left(\lambda-L_{b c}^{0}\right)^{-1}$ is well defined for $\lambda \notin S p\left(L_{b c}^{0}\right)$ by

$$
R_{b c}^{0}(\lambda) \varphi_{k}^{\mu}=\frac{1}{\lambda-\tau_{*}-k} \varphi_{k}^{\mu}, \quad k \in 2 \mathbb{Z}, \quad \mu=1,2 .
$$

The standard perturbation formula for the resolvent

$$
R_{b c}(\lambda)=R_{b c}^{0}(\lambda)+R_{b c}^{0}(\lambda) V R_{b c}^{0}(\lambda)+R_{b c}^{0}(\lambda) V R_{b c}^{0}(\lambda) V R_{b c}^{0}(\lambda)+\cdots
$$

can be written as

$$
R_{b c}(\lambda)=\left(K_{\lambda}\right)^{2}+\sum_{s=1}^{\infty} K_{\lambda}\left(K_{\lambda} V K_{\lambda}\right)^{s} K_{\lambda}
$$

provided

$$
\left(K_{\lambda}\right)^{2}=R_{b c}^{0}(\lambda)
$$

Then the operator $R_{b c}(\lambda)$ is well-defined by (4.8) if

$$
\left\|K_{\lambda} V K_{\lambda}\right\|<1 \text {. }
$$

In the next section we will give conditions under which (4.10) holds.

In view of (4.6) and (4.7), we define an operator $K=K_{\lambda}$ with the property (4.9), respectively, for strictly regular $b c$ by

$$
K_{\lambda} \varphi_{k}^{\mu}=\frac{1}{\sqrt{\lambda-\tau_{\mu}-k}} \varphi_{k}^{\mu}, \quad k \in 2 \mathbb{Z}, \quad \mu=1,2,
$$

and for regular but not strictly regular $b c$ by

$$
K_{\lambda} \varphi_{k}^{\mu}=\frac{1}{\sqrt{\lambda-\tau_{*}-k}} \varphi_{k}^{\mu}, \quad k \in 2 \mathbb{Z}, \quad \mu=1,2,
$$

where

$$
\sqrt{z}=\sqrt{r} e^{i \varphi / 2} \quad \text { if } \quad z=r e^{i \varphi}, \quad-\pi \leq \varphi<\pi .
$$

By (4.1), (4.2), (4.11) and (4.12), we have

$$
\left\langle K_{\lambda} V K_{\lambda} \varphi_{k}^{\nu}, \tilde{\varphi}_{j}^{\mu}\right\rangle=\frac{w^{\mu \nu}(j+k)}{\sqrt{\lambda-\tau_{\mu}-j} \sqrt{\lambda-\tau_{\nu}-k}}, \quad j, k \in 2 \mathbb{Z}
$$


for strictly regular $b c$, and

$$
\left\langle K_{\lambda} V K_{\lambda} \varphi_{k}^{\nu}, \tilde{\varphi}_{j}^{\mu}\right\rangle=\frac{w^{\mu \nu}(j+k)}{\sqrt{\lambda-\tau_{*}-j} \sqrt{\lambda-\tau_{*}-k}}, \quad j, k \in 2 \mathbb{Z}
$$

for regular but not strictly regular $b c$.

Therefore, for $s \geq 1$, it follows that

$$
\left\langle K_{\lambda}\left(K_{\lambda} V K_{\lambda}\right)^{s} K_{\lambda} \varphi_{k}^{\nu}, \tilde{\varphi}_{m}^{\mu}\right\rangle
$$

$=\sum_{\gamma_{1}, . ., \gamma_{s}=1}^{2} \sum_{j_{1}, \ldots j_{s}} \frac{w^{\mu \gamma_{1}}\left(m+i_{1}\right) w^{\gamma_{1} \gamma_{2}}\left(i_{1}+i_{2}\right) \cdots w^{\gamma_{s-1} \gamma_{s}}\left(i_{s-1}+i_{s}\right) w^{\gamma_{s} \nu}\left(i_{s}+k\right)}{\left(\lambda-\tau_{\mu}-m\right)\left(\lambda-\tau_{\gamma_{1}}-i_{1}\right) \cdots\left(\lambda-\tau_{\gamma_{s}}-i_{s}\right)\left(\lambda-\tau_{\nu}-k\right)}$

for strictly regular $b c$, and

$$
\left\langle K_{\lambda}\left(K_{\lambda} V K_{\lambda}\right)^{s} K_{\lambda} \varphi_{k}^{\nu}, \tilde{\varphi}_{j}^{\mu}\right\rangle
$$

$=\sum_{\gamma_{1}, . ., \gamma_{s}=1}^{2} \sum_{j_{1}, \ldots j_{s}} \frac{w^{\mu \gamma_{1}}\left(m+i_{1}\right) w^{\gamma_{1} \gamma_{2}}\left(i_{1}+i_{2}\right) \cdots w^{\gamma_{s-1} \gamma_{s}}\left(i_{s-1}+i_{s}\right) w^{\gamma_{s} \nu}\left(i_{s}+k\right)}{\left(\lambda-\tau_{*}-m\right)\left(\lambda-\tau_{*}-i_{1}\right) \cdots\left(\lambda-\tau_{*}-i_{s}\right)\left(\lambda-\tau_{*}-k\right)}$.

for regular but not strictly regular bc. In view of (4.8), the formulas (4.15) and (4.16) determine the matrix representation of the resolvent $R_{b c}(\lambda)$.

\section{LOCALIZATION OF SPECTRA}

In this section we consider the spectra localization of the operators $L_{b c}=L_{b c}^{0}+V$, where $V$ denotes the operator of multiplication by the matrix $v(x)=\left(\begin{array}{cc}0 & P(x) \\ Q(x) & 0\end{array}\right)$.

1. In view of (4.13) and (4.14), the Hilbert-Schmidt norm of the operator $K_{\lambda} V K_{\lambda}$ with respect to the Riesz basis $\Phi$ (see (2.5)) is given by

$$
\left(\left\|K_{\lambda} V K_{\lambda}\right\|_{H S}^{*}\right)^{2}=\sum_{\nu, \mu=1}^{2} \sum_{j, k \in 2 \mathbb{Z}} \frac{\left|w^{\mu \nu}(j+k)\right|^{2}}{\left|\lambda-\tau_{\mu}-j\right|\left|\lambda-\tau_{\nu}-k\right|}
$$

for regular $b c$, and

$$
\left(\left\|K_{\lambda} V K_{\lambda}\right\|_{H S}^{*}\right)^{2}=\sum_{\nu, \mu=1}^{2} \sum_{j, k \in 2 \mathbb{Z}} \frac{\left|w^{\mu \nu}(j+k)\right|^{2}}{\left|\lambda-\tau_{*}-j\right|\left|\lambda-\tau_{*}-k\right|}
$$

for regular but not strictly regular $b c$.

For convenience, we set

$$
r(m)=\max \left\{\left|w^{\mu \nu}(m)\right|, \mu, \nu=1,2\right\}, \quad m \in 2 \mathbb{Z} ;
$$


then

$$
r=(r(k)) \in \ell^{2}(2 \mathbb{Z}), \quad\|r\| \leq C\left(\|P\|_{L^{2}}+\|Q\|_{L^{2}}\right),
$$

where $C=C(b c)$.

Now we define operators $\bar{V}$ and $\bar{K}_{\lambda}$ which matrix representations dominate, respectively, the matrix representations of $V$ and $K_{\lambda}$, as follows:

$$
\begin{gathered}
\bar{V} \varphi_{n}^{\mu}=\sum_{k \in 2 \mathbb{Z}} r(k+n)\left(\varphi_{k}^{1}+\varphi_{k}^{2}\right), \quad \mu=1,2 ; n \in 2 \mathbb{Z}, \\
\bar{K}_{\lambda} \varphi_{n}^{\mu}=\frac{1}{\sqrt{\left|\lambda-\tau_{\mu}-n\right|}} \varphi_{n}^{\mu}, \quad \mu=1,2 ; \quad n \in 2 \mathbb{Z}
\end{gathered}
$$

for strictly regular $b c$, and

$$
\bar{K}_{\lambda} \varphi_{n}^{\mu}=\frac{1}{\sqrt{\left|\lambda-\tau_{*}-n\right|}} \varphi_{n}^{\mu}, \quad \mu=1,2 ; \quad n \in 2 \mathbb{Z}
$$

for regular but not strictly regular $b c$.

The matrix elements of the operator $K_{\lambda} V K_{\lambda}$ do not exceed, by absolute value, the matrix elements of $\bar{K}_{\lambda} \bar{V} \bar{K}_{\lambda}$. Therefore, in view of (5.1)

- (5.3) and Lemma 9, it follows that

$$
\left(\left\|K_{\lambda} V K_{\lambda}\right\|_{H S}^{*}\right)^{2} \leq\left(\left\|\bar{K}_{\lambda} \bar{V} \bar{K}_{\lambda}\right\|_{H S}^{*}\right)^{2}=\sum_{\mu, \nu=1}^{2} \sum_{j, k \in 2 \mathbb{Z}} \frac{|r(j+k)|^{2}}{\left|\lambda-\tau_{\mu}-j\right|\left|\lambda-\tau_{\nu}-k\right|},
$$

for regular $b c$, and

$$
\left(\left\|K_{\lambda} V K_{\lambda}\right\|_{H S}^{*}\right)^{2} \leq\left(\left\|\bar{K}_{\lambda} \bar{V} \bar{K}_{\lambda}\right\|_{H S}^{*}\right)^{2}=4 \sum_{j, k \in 2 \mathbb{Z}} \frac{|r(j+k)|^{2}}{\left|\lambda-\tau_{*}-j\right|\left|\lambda-\tau_{*}-k\right|},
$$

for regular but not strictly regular $b c$.

For each $\ell^{2}$-sequence $x=(x(j))_{j \in \mathbb{Z}}$ and $m \in \mathbb{N}$ we set

$$
\mathcal{E}_{m}(x)=\left(\sum_{|j| \geq m}|x(j)|^{2}\right)^{1 / 2}
$$

Next we consider separately the case of strictly regular $b c$ and the case of regular but not strictly regular $b c$.

2. Strictly regular bc. We subdivide the complex plane $\mathbb{C}$ into strips (5.11)

$$
H_{m}=\left\{z \in \mathbb{C}:-1 \leq \operatorname{Re}\left(z-m-\frac{\tau_{1}+\tau_{2}}{2}\right) \leq 1\right\}, \quad m \in 2 \mathbb{Z},
$$


and set

$$
\begin{gathered}
H^{N}=\bigcup_{|m| \leq N} H_{m} \\
R_{N T}=\left\{z=x+i t:\left|x-\operatorname{Re} \frac{\tau_{1}+\tau_{2}}{2}\right|<N+1,|t|<T\right\},
\end{gathered}
$$

where $N \in 2 \mathbb{N}$ and

$$
T=2 \max \left(\left|\operatorname{Im} \tau_{1}\right|,\left|\operatorname{Im} \tau_{2}\right|, 384\|A\|\left\|A^{-1}\right\|\|r\|^{2}\right)
$$

with $A$ being the isomorphism defined by (3.29).

Let

$$
\rho:=\min \left(1-\left|\operatorname{Re}\left(\tau_{1}-\tau_{2}\right)\right| / 2,\left|\tau_{1}-\tau_{2}\right| / 2\right),
$$

and

$$
D_{m}^{\mu}=\left\{z \in \mathbb{C}:\left|z-\tau_{\mu}-m\right|<\rho\right\}, \quad m \in 2 \mathbb{Z} .
$$

Lemma 10. (a) In the above notations, the following estimates hold:

(a) if $\lambda \in H_{m} \backslash\left(D_{m}^{1} \cup D_{m}^{2}\right), m \neq 0$, then

$$
\sum_{\mu, \nu=1}^{2} \sum_{j, k \in 2 \mathbb{Z}} \frac{|r(j+k)|^{2}}{\left|\lambda-\tau_{\mu}-j\right|\left|\lambda-\tau_{\nu}-k\right|} \leq\left(\frac{30}{\rho}\right)^{2}\left(\frac{\|r\|^{2}}{\sqrt{|m|}}+\left(\mathcal{E}_{|m|}(r)\right)^{2}\right) ;
$$

(b) if $\lambda \in H^{N} \backslash R_{N T}$, then

$$
\sum_{\mu, \nu=1}^{2} \sum_{j, k \in 2 \mathbb{Z}} \frac{|r(j+k)|^{2}}{\left|\lambda-\tau_{\mu}-j\right|\left|\lambda-\tau_{\nu}-k\right|} \leq \frac{384}{T}\|r\|^{2}
$$

Proof. (a) If $\lambda \in H_{m}$ then

$$
\left|\lambda-\tau_{\mu}-j\right| \geq|m-j| / 4, \quad j \in 2 \mathbb{Z} \backslash\{m\}, \quad \mu=1,2 .
$$

Indeed, $|m-j| \geq 2$, so (5.11) and (3.21) imply $\left|R e\left(\lambda-\tau_{\mu}-j\right)\right| \geq|m-j|-1-\frac{1}{2}\left|\operatorname{Re}\left(\tau_{1}-\tau_{2}\right)\right| \geq|m-j|-\frac{3}{2} \geq \frac{1}{4}|m-j|$.

In view of (5.19), the sum in (5.17) does not exceed

$$
4^{3} \sum_{j, k \neq m} \frac{\mid r\left(j+\left.k\right|^{2}\right.}{|m-j||m-k|}+4^{2} \sum_{k \neq m} \frac{\mid r\left(m+\left.k\right|^{2}\right.}{\rho|m-k|}+4^{2} \sum_{j \neq m} \frac{\mid r\left(j+\left.m\right|^{2}\right.}{|m-j| \rho}+4 \frac{|r(2 m)|^{2}}{\rho^{2}} \text {. }
$$

Now the estimate (5.17) follows from the inequalities (5.20) and (5.21) below. 
Lemma 11. If $r=(r(k)) \in \ell^{2}(2 \mathbb{Z})$, then

$$
\begin{gathered}
\sum_{k \neq n} \frac{|r(n+k)|^{2}}{|n-k|} \leq \frac{\|r\|^{2}}{|n|}+\left(\mathcal{E}_{|n|}(r)\right)^{2}, \quad|n| \geq 1 \\
\sum_{i, k \neq n} \frac{|r(i+k)|^{2}}{|n-i||n-k|} \leq 12\left(\frac{\|r\|^{2}}{\sqrt{|n|}}+\left(\mathcal{E}_{|n|}(r)\right)^{2}\right), \quad|n| \geq 1,
\end{gathered}
$$

Lemma 11 is identical to Lemma 7 in 9 ; a proof is provided there.

Next we prove (5.18). If $\lambda \in H^{N} \backslash R_{N T}$, then $\lambda \in H_{m}$ for some even integer $m \in[-N, N]$, and we have

$$
\left|\lambda-\tau_{\mu}-j\right| \geq \frac{1}{4 \sqrt{2}}(|j-m|+T), \quad \mu=1,2 ; j \in 2 \mathbb{Z} .
$$

Indeed, $\lambda \in H_{m} \backslash R_{N T}$ means that

$$
\lambda=m+R e \frac{\tau_{1}+\tau_{2}}{2}+\xi+i \eta \quad \text { with } \xi, \eta \in \mathbb{R}, \quad|\xi| \leq 1,|\eta| \geq T .
$$

Therefore, if $j=m$, then by (5.14) we obtain

$$
\left|\lambda-\tau_{\mu}-j\right| \geq\left|\operatorname{Im}\left(\lambda-\tau_{\mu}-j\right)\right| \geq T-\left|\operatorname{Im} \tau_{\mu}\right| \geq T / 2,
$$

so (15.22) holds. Otherwise, $|j-m| \geq 2$ (so $|j-m|-3 / 2 \geq|j-m| / 4)$; then by the inequality $|x+i y| \geq \frac{1}{\sqrt{2}}|x|+\frac{1}{\sqrt{2}}|y|$ and (3.21) we obtain

$$
\begin{gathered}
\left|\lambda-\tau_{\mu}-j\right| \geq \frac{1}{\sqrt{2}}\left(|j-m|-\left|\operatorname{Re} \frac{\tau_{1}-\tau_{2}}{2}\right|-1\right)+\frac{1}{\sqrt{2}}\left(T-\left|I m \tau_{\mu}\right|\right) \\
\geq \frac{1}{\sqrt{2}}(|j-m|-3 / 2)+\frac{1}{2 \sqrt{2}} T \geq \frac{1}{4 \sqrt{2}}(|j-m|+T) .
\end{gathered}
$$

In view of (5.22), the sum in (5.18) does not exceed

$$
\sigma:=128 \sum_{j, k \in 2 \mathbb{Z}} \frac{|r(j+k)|^{2}}{(|j-m|+T)(|k-m|+T)} .
$$

By the Cauchy inequality,

$$
\sigma \leq 128\left(\sum_{j, k \in 2 \mathbb{Z}} \frac{|r(j+k)|^{2}}{(|j-m|+T)^{2}}\right)^{1 / 2}\left(\sum_{j, k \in 2 \mathbb{Z}} \frac{|r(j+k)|^{2}}{(|k-m|+T)^{2}}\right)^{1 / 2} .
$$

Since

$$
\sum_{j \in 2 \mathbb{Z}} \frac{1}{(|j-m|+T)^{2}} \leq \frac{1}{T^{2}}+2 \int_{0}^{\infty} \frac{1}{(x+T)^{2}} d x=\frac{1}{T^{2}}+\frac{2}{T} \leq \frac{3}{T},
$$

it follows that $\sigma \leq \frac{384}{T}\|r\|^{2}$, which completes the proof. 
Theorem 12. In the above notations, for each strictly regular bc there is an $N=N(v, b c) \in 2 \mathbb{N}$ such that

$$
S p\left(L_{b c}\left(v_{\zeta}\right) \subset R_{N T} \cup \bigcup_{|m|>N}\left(D_{m}^{1} \cup D_{m}^{2}\right) \quad \text { for } v_{\zeta}=\zeta v,|\zeta| \leq 1 .\right.
$$

Proof. Let $G$ be the set in the right-hand side of (5.23). In order to prove (5.23) for $\zeta=1$, it is enough to explain that the resolvent $R_{\lambda}(v)=(\lambda-L(v))^{-1}$ is well-defined for $\lambda \in \mathbb{C} \backslash G$.

In view of (4.8) - (4.10), $R_{\lambda}(v)$ is well-defined if $\left\|K_{\lambda} V K_{\lambda}\right\|<1$. From Lemma 1, formula (5.8), Lemma 10 and the choice (5.14) of the constant $T$ it follows that

$\left\|K_{\lambda} V K_{\lambda}\right\| \leq\left\|K_{\lambda} V K_{\lambda}\right\|_{H S} \leq\|A\|\left\|A^{-1}\right\|\left\|K_{\lambda} V K_{\lambda}\right\|_{H S}^{*}<1$ for $\lambda \in \mathbb{C} \backslash G$

if $N$ is chosen so large that the right-hand sides of (5.17) (for $|m|>N$ ) and (5.18) are strictly less than 1 . In view of Lemma 9] and (5.3), (5.24) holds for $\zeta v,|\zeta| \leq 1$ as well. Therefore, (5.23) holds with $N=N(v, b c)$.

3. Regular but not strictly regular boundary conditions. Now we subdivide the complex plane $\mathbb{C}$ into strips

$$
H_{m}=\left\{z \in \mathbb{C}:-1 \leq \operatorname{Re}\left(z-m-\tau_{*}\right) \leq 1\right\}, \quad m \in 2 \mathbb{Z},
$$

and set

$$
\begin{gathered}
H^{N}=\bigcup_{|m| \leq N} H_{m}, \\
R_{N T}=\left\{z=x+i t:\left|x-\operatorname{Re} \tau_{*}\right|<N+1,|t|<T\right\},
\end{gathered}
$$

where $N \in 2 \mathbb{N}$ and

$$
T=2 \max \left(\left|I m \tau_{*}\right|, 96\|A\|\left\|A^{-1}\right\|\|r\|^{2}\right)
$$

with $A$ being the isomorphism defined by (3.29) (for periodic type boundary conditions) and (3.59) otherwise.

Let

$$
D_{m}=\left\{z \in \mathbb{C}:\left|z-\tau_{*}-m\right|<1 / 4\right\}, \quad m \in 2 \mathbb{Z} .
$$

Lemma 13. (a) In the above notations, if $\left.\lambda \in H_{m} \backslash D_{m}\right), m \neq 0$, then

$$
\sum_{j, k \in 2 \mathbb{Z}} \frac{|r(j+k)|^{2}}{\left|\lambda-\tau_{*}-j\right|\left|\lambda-\tau_{*}-k\right|} \leq C\left(\frac{\|r\|^{2}}{\sqrt{|m|}}+\left(\mathcal{E}_{|m|}(r)\right)^{2}\right),
$$

where $C$ is an absolute constant; 
(b) if $\lambda \in H^{N} \backslash R_{N T}$, then

$$
\sum_{j, k \in 2 \mathbb{Z}} \frac{|r(j+k)|^{2}}{\left|\lambda-\tau_{*}-j \| \lambda-\tau_{*}-k\right|} \leq \frac{24}{T}\|r\|^{2} .
$$

Proof. If $\lambda \in H_{m}$ then (compare with (5.19))

$$
\left|\lambda-\tau_{*}-j\right| \geq|m-j| / 4 \quad j \neq m, j \in \mathbb{Z} .
$$

Therefore, the sum in (5.30) does not exceed

$4^{2} \sum_{j, k \neq m} \frac{\mid r\left(j+\left.k\right|^{2}\right.}{|m-j||m-k|}+4^{2} \sum_{k \neq m} \frac{\mid r\left(j+\left.k\right|^{2}\right.}{|m-k|}+4^{2} \sum_{j \neq m} \frac{\mid r\left(j+\left.k\right|^{2}\right.}{|m-j|}+4^{2}|r(2 m)|^{2}$.

Now the estimate (5.30) follows from the inequalities (5.20) and (5.21) in Lemma 11.

Next we prove (5.31). If $\lambda \in H^{N} \backslash R_{N T}$, then $\lambda \in H_{m}$ for some integer $m \in[-N, N]$; then (compare with (15.22) ) we have

$$
\left|\lambda-\tau_{*}-j\right| \geq \frac{1}{2 \sqrt{2}}(|j-m|+T), \quad \mu=1,2 ; j \in 2 \mathbb{Z} .
$$

The proof of (5.33) is similar to the proof of (5.22), and therefore, it is omitted. Moreover, using (5.33) one can complete the proof of part (b) exactly as it is done in the proof of Lemma 10.

Theorem 14. In the above notations, for each regular but not strictly regular bc there is $N=N(v, b c) \in 2 \mathbb{N}$ such that

$$
S p\left(L_{b c}\left(v_{\zeta}\right) \subset R_{N T} \cup \bigcup_{|m|>N} D_{m} \quad \text { for } v_{\zeta}=\zeta v,|\zeta| \leq 1 .\right.
$$

Proof. We follow the proof of Theorem 12 but use instead of (15.8), Lemma 10 and (5.14) their counterparts (5.9), Lemma 13 and (5.28). We omit further details.

\section{BARI-MARKUS PROPERTY IN THE CASE OF STRICTLY REGULAR BOUNDARY CONDITIONS}

We use the notations of the previous section. For strictly regular $b c$ Theorem 12 gives the following localization of the spectrum of the Dirac operator $L_{b c}$ :

$$
S p\left(L_{b c}\right) \subset R_{N T} \cup \bigcup_{|n|>N}\left(D_{n}^{1} \cup D_{n}^{2}\right) .
$$


Let us consider the Riesz projections associated with $L_{b c}$ (6.1)

$S_{N}=\frac{1}{2 \pi i} \int_{\partial R_{N T}}(\lambda-L)^{-1} d \lambda, \quad P_{n, \alpha}=\frac{1}{2 \pi i} \int_{\partial D_{n}^{\alpha}}(\lambda-L)^{-1} d \lambda, \quad \alpha=1,2$,

and let $S_{N}^{0}$ and $P_{n, \alpha}^{0}$ be the Riesz projections associated with the free operator $L_{b c}^{0}$.

Theorem 15. Suppose $L_{b c}$ and $L_{b c}^{0}$ are, respectively, the Dirac operator with an $L^{2}$ potential $v$ and the corresponding free Dirac operator, subject to the same strictly regular boundary conditions bc. Then, there is an $N \in 2 \mathbb{N}$ such that the Riesz projections $S_{N}, P_{n, \alpha}$ and $S_{N}^{0}, P_{n, \alpha}^{0}, n \in$ $2 \mathbb{Z},|n|>N, \alpha=1,2$, associated with $L$ and $L^{0}$ are well defined by (6.1), and we have

$$
\begin{gathered}
\operatorname{dim} P_{n, \alpha}=\operatorname{dim} P_{n, \alpha}^{0}=1, \quad \operatorname{dim} S_{N}=\operatorname{dim} S_{N}^{0}=2 N ; \\
\sum_{|n|>N}\left\|P_{n, \alpha}-P_{n, \alpha}^{0}\right\|^{2}<\infty, \quad \alpha=1,2 .
\end{gathered}
$$

Moreover, the system $\left\{S_{N}, P_{n, \alpha}, n \in 2 \mathbb{Z},|n|>N, \alpha=1,2\right\}$ is a Riesz basis of projections in $L^{2}\left([0, \pi], \mathbb{C}^{2}\right)$, i.e.,

$$
\mathbf{f}=S_{N}(\mathbf{f})+\sum_{\alpha=1}^{2} \sum_{|n|>N} P_{n, \alpha}(\mathbf{f}) \quad \forall \mathbf{f} \in L^{2}\left([0, \pi], \mathbb{C}^{2}\right),
$$

where the series converge unconditionally.

Proof. In view of Theorem [12, there is an $N=N(v, b c)$ such that the projections

$S_{N}(\zeta)=\frac{1}{2 \pi i} \int_{\partial R_{N T}}(\lambda-L(\zeta v))^{-1} d \lambda, \quad P_{n, \alpha}(\zeta)=\frac{1}{2 \pi i} \int_{\partial D_{n}^{\alpha}}(\lambda-L(\zeta v))^{-1} d \lambda$,

$|n|>N, \alpha=1,2$, are well-defined for $|\zeta| \leq 1$ and depend continuously (even analytically) on $\zeta$. Therefore, their dimensions

$$
\operatorname{dim} S_{N}(\zeta)=\operatorname{trace} S_{N}(\zeta), \quad \operatorname{dim} P_{n, \alpha}(\zeta)=\operatorname{trace} P_{n, \alpha}(\zeta)
$$

are constants as continuous integer-valued functions. This proves (6.2).

Next we prove (6.3). For periodic, antiperiodic and Dirichlet boundary conditions (6.3) was proved in [9, Theorem 3]; here we follow the same approach.

For large enough $N$ the series in (4.8) converges (see formula (5.24) the proof of Theorem 12); therefore,

$$
P_{n, \alpha}-P_{n, \alpha}^{0}=\frac{1}{2 \pi i} \int_{\partial D_{n}^{\alpha}} \sum_{s=0}^{\infty} K_{\lambda}\left(K_{\lambda} V K_{\lambda}\right)^{s+1} K_{\lambda} d \lambda .
$$


Let $\Phi=\left\{\varphi_{k}^{1}, \varphi_{k}^{2}, k \in \mathbb{Z}\right\}$ and $\tilde{\Phi}=\left\{\tilde{\varphi}_{k}^{1}, \tilde{\varphi}_{k}^{2}, k \in \mathbb{Z}\right\}$ be the Riesz basis (consisting of eigenfunctions of the operator $L_{b c}^{0}$ ) and its biorthogonal system that are constructed in Lemma 5. We are going to prove (6.3) by estimating the Hilbert-Schmidt norms $\left\|P_{n, \alpha}-P_{n, \alpha}^{0}\right\|_{H S}^{*}$ with respect to the basis $\Phi$.

Recall that

$$
\left(\left\|P_{n, \alpha}-P_{n, \alpha}^{0}\right\|_{H S}^{*}\right)^{2}=\sum_{\mu, \nu=1}^{2} \sum_{m, k \in 2 \mathbb{Z}}\left|\left\langle\left(P_{n, \alpha}-P_{n, \alpha}^{0}\right) \varphi_{m}^{\mu}, \tilde{\varphi}_{k}^{\nu}\right\rangle\right|^{2} .
$$

By (6.5), we obtain

$$
\left\langle\left(P_{n, \alpha}-P_{n, \alpha}^{0}\right) \varphi_{m}^{\mu}, \tilde{\varphi}_{k}^{\nu}\right\rangle=\sum_{s=0}^{\infty} I_{n, \alpha}^{\nu, \mu}(s, k, m)
$$

where

$$
I_{n, \alpha}^{\nu, \mu}(s, k, m)=\frac{1}{2 \pi i} \int_{\partial D_{n}^{\alpha}}\left\langle K_{\lambda}\left(K_{\lambda} V K_{\lambda}\right)^{s+1} K_{\lambda} \varphi_{m}^{\mu}, \tilde{\varphi}_{k}^{\nu}\right\rangle d \lambda .
$$

Therefore,

$$
\sum_{|n|>N}\left(\left\|P_{n, \alpha}-P_{n, \alpha}^{0}\right\|_{H S}^{*}\right)^{2} \leq \sum_{s, t=0}^{\infty} \sum_{|n|>N} \sum_{\mu, \nu=1}^{2} \sum_{m, k \in \mathbb{Z}}\left|I_{n, \alpha}^{\nu, \mu}(s, k, m)\right| \cdot\left|I_{n, \alpha}^{\nu, \mu}(t, k, m)\right| .
$$

Now, the Cauchy inequality implies

$$
\sum_{|n|>N}\left(\left\|P_{n}-P_{n}^{0}\right\|_{H S}^{*}\right)^{2} \leq \sum_{s, t=0}^{\infty}(A(s))^{1 / 2}(A(t))^{1 / 2}
$$

where

$$
A(s)=\sum_{|n|>N} \sum_{\mu, \nu=1}^{2} \sum_{m, k \in \mathbb{Z}}\left|I_{n, \alpha}^{\nu, \mu}(s, k, m)\right|^{2} .
$$

Of course, $A(s)$ depends on $\alpha$ and $N$ but that dependence is suppressed in the notation.

In view of (4.15) and (6.6), it follows that

$$
\begin{gathered}
I_{n, \alpha}^{\nu, \mu}(s, k, m)=\frac{1}{2 \pi i} \int_{\partial D_{n}^{\alpha}} \sum_{\gamma_{1}, . ., \gamma_{s}=1}^{2} \sum_{j_{1}, \ldots j_{s}} \frac{w^{\nu \gamma_{1}}\left(k+j_{1}\right)}{\left(\lambda-\tau_{\nu}-k\right)} \times \\
\times \frac{w^{\gamma_{1} \gamma_{2}}\left(j_{1}+j_{2}\right) \cdots w^{\gamma_{s-1} \gamma_{s}}\left(j_{s-1}+j_{s}\right) w^{\gamma_{s} \mu}\left(j_{s}+m\right)}{\left(\lambda-\tau_{\gamma_{1}}-j_{1}\right) \cdots\left(\lambda-\tau_{\gamma_{s}}-j_{s}\right)\left(\lambda-\tau_{\mu}-m\right)} d \lambda .
\end{gathered}
$$


By the Cauchy formula, if $n \notin\left\{k, j_{1}, \ldots, j_{s}, m\right\}$ then (6.10)

$$
\int_{\partial D_{n}^{\alpha}} \frac{w^{\nu \gamma_{1}}\left(k+j_{1}\right) w^{\gamma_{1} \gamma_{2}}\left(j_{1}+j_{2}\right) \cdots w^{\gamma_{s} \mu}\left(j_{s}+m\right)}{\left(\lambda-\tau_{\nu}-k\right)\left(\lambda-\tau_{\gamma_{1}}-j_{1}\right) \cdots\left(\lambda-\tau_{\gamma_{s}}-j_{s}\right)\left(\lambda-\tau_{\mu}-m\right)} d \lambda=0 .
$$

This observation is crucial for the proof. We remove from the sum in (6.9) the terms which integrals are zeros and after that estimate the remaining terms by absolute value as follows.

Let $r$ be the $\ell^{2}(2 \mathbb{Z})$-sequence defined in (5.3). We set

$$
B\left(z, k, j_{1}, \ldots, j_{s}, m\right)=\frac{r\left(k+j_{1}\right) r\left(j_{1}+j_{2}\right) \cdots r\left(j_{s-1}+j_{s}\right) r\left(j_{s}+m\right)}{|z-k|\left|z-j_{1}\right| \cdots\left|z-j_{s}\right||z-m|}
$$

for $s>0$, and

$$
B(z, k, m)=\frac{r(m+k)}{|z-k||z-m|}
$$

in the case when $s=0$ and there are no $j$-indices.

Lemma 16. In the above notations, we have

$$
A(s) \leq 4 \rho(2 C)^{s}\left(B_{1}(s)+B_{2}(s)+B_{3}(s)+B_{4}(s)\right),
$$

with $C=C(\rho)$ and

$$
B_{1}(s)=\sum_{|n|>N} \sup _{|z-n|=\rho}\left(\sum_{j_{1}, \ldots, j_{s}} B\left(z, n, j_{1}, \ldots, j_{s}, n\right)\right)^{2} ;
$$

$$
B_{2}(s)=\sum_{|n|>N} \sum_{k \neq n} \sup _{|z-n|=\rho}\left(\sum_{j_{1}, \ldots, j_{s}} B\left(z, k, j_{1}, \ldots, j_{s}, n\right)\right)^{2}
$$

$$
B_{3}(s)=\sum_{|n|>N} \sum_{m \neq n} \sup _{|z-n|=\rho}\left(\sum_{j_{1}, \ldots, j_{s}} B\left(z, n, j_{1}, \ldots, j_{s}, m\right)\right)^{2}
$$

$$
B_{4}(s)=\sum_{|n|>N} \sum_{m, k \neq n} \sup _{|z-n|=\rho}\left(\sum_{j_{1}, \ldots, j_{s}}^{*} B\left(z, k, j_{1}, \ldots, j_{s}, m\right)\right)^{2}, \quad s \geq 1,
$$

where the symbol * over the sum in the parentheses means that at least one of the indices $j_{1}, \ldots, j_{s}$ is equal to $n$.

Proof. In view of (6.8), we have

$$
A(s) \leq A_{1}(s)+A_{2}(s)+A_{3}(s)+A_{4}(s),
$$


where

$$
\begin{aligned}
& A_{1}(s)=\sum_{|n|>N} \sum_{\nu, \mu=1}^{2}\left|I_{n, \alpha}^{\nu, \mu}(s, n, n)\right|^{2}, \quad A_{2}(s)=\sum_{|n|>N} \sum_{\nu, \mu=1}^{2} \sum_{k \neq n}\left|I_{n, \alpha}^{\nu, \mu}(s, k, n)\right|^{2}, \\
& A_{3}(s)=\sum_{|n|>N} \sum_{\nu, \mu=1}^{2} \sum_{m \neq n}\left|I_{n, \alpha}^{\nu, \mu}(s, n, m)\right|^{2}, \quad A_{4}(s)=\sum_{|n|>N} \sum_{\nu, \mu=1}^{2} \sum_{k, m \neq n}\left|I_{n, \alpha}^{\nu, \mu}(s, k, m)\right|^{2} .
\end{aligned}
$$

So, the lemma will be proved if we show that $A_{i}(s) \leq 4 \rho(2 C)^{s} B_{i}(s)$, $i=1,2,3,4$.

If $\lambda \in \partial D_{n}^{\alpha}$ and $z=\lambda-\tau_{\alpha}$, then we have

$$
\begin{aligned}
& \left|\frac{w^{\nu \gamma_{1}}\left(k+j_{1}\right) w^{\gamma_{1} \gamma_{2}}\left(j_{1}+j_{2}\right) \cdots w^{\gamma_{s-1} \gamma_{s}}\left(j_{s-1}+j_{s}\right) w^{\gamma_{s} \mu}\left(j_{s}+m\right)}{\left(\lambda-\tau_{\nu}-k\right)\left(\lambda-\tau_{\gamma_{1}}-j_{1}\right) \cdots\left(\lambda-\tau_{\gamma_{s}}-j_{s}\right)\left(\lambda-\tau_{\mu}-m\right)}\right| \\
& \leq C^{s} B\left(z, k, j_{1}, \ldots, j_{s}, m\right), \quad \text { where } C=C(\rho)>1 .
\end{aligned}
$$

In order to prove (6.18) it is enough to show that

$$
\left|z+\tau_{\alpha}-\tau_{\beta}-j\right| \geq \frac{1}{C}|z-j|, \quad \text { if } \quad|z-n|=\rho, \quad \beta \neq \alpha .
$$

If $j=n$, then by the choice of $\rho$ in (5.15) we have

$$
\left|z+\tau_{\alpha}-\tau_{\beta}-n\right| \geq\left|\tau_{1}-\tau_{2}\right|-|z-n|=\left|\tau_{1}-\tau_{2}\right|-\rho \geq \rho=|z-n| .
$$

Otherwise, $|n-j| \geq 2$, so taking into account that $\left|\operatorname{Re}\left(\tau_{\alpha}-\tau_{\beta}\right)\right| \leq 1$ due to (3.21), we obtain

$$
\left|z+\tau_{\alpha}-\tau_{\beta}-j\right| \geq|n-j|-\rho-\left|\operatorname{Re}\left(\tau_{\alpha}-\tau_{\beta}\right)\right| \geq|n-j|-\rho-1 .
$$

Since $|z-j| \leq|n-j|+\rho$, it is enough to find a constant $C$ such that

$$
|n-j|-\rho-1 \geq \frac{1}{C}(|n-j|+\rho),
$$

or equivalently, $(C-1)|n-j| \geq(C+1) \rho+C$. For $|n-j|=2$ the latter inequality is equivalent to $C(1-\rho) \geq 2+\rho$. Therefore, (6.19) holds with $C=C(\rho)=(2+\rho) /(1-\rho)$.

Now, (6.9) and (6.18) imply that

$$
\left|I_{n, \alpha}^{\nu, \mu}(s, k, m)\right| \leq \rho(2 C)^{s} \sup _{|z-n|=\rho} \sum_{j_{1}, \ldots, j_{s}} B\left(z, k, j_{1}, \ldots, j_{s}, m\right),
$$

where $C=C(\rho)$ is the constant from (6.18). Therefore, in view of (6.14) - (6.16), we obtain

$$
A_{i}(s) \leq 4 \rho(2 C)^{s} B_{i}(s), \quad i=1,2,3 .
$$

Finally, taking into account (6.10) we remove from the sum in the right-hand side of (6.20) the terms associated with sets indices $k, j_{1}, \ldots, j_{s}, m$ 
such that $n \notin\left\{k, j_{1}, \ldots, j_{s}, m\right\}$. This leads to the following improvement of (6.20):

$$
\left|I_{n, \alpha}^{\nu, \mu}(s, k, m)\right| \leq \rho(2 C)^{s} \sup _{|z-n|=\rho} \sum_{j_{1}, \ldots, j_{s}}^{*} B\left(z, k, j_{1}, \ldots, j_{s}, m\right), \quad k, m \neq n .
$$

In view of (6.17), this yields $A_{4}(s) \leq 4 \rho(2 C)^{s} B_{4}(s)$, which completes the proof.

Proposition 17. In the above notations, $(6.21)$

$$
B_{\nu}(s) \leq C_{1}\|r\|^{2} a_{N}^{2 s}, \quad \nu=1,2,3, \quad B_{4}(s) \leq C_{1} s\|r\|^{4} a_{N}^{2(s-1)}, s \geq 1,
$$

where

$$
a_{N}=\frac{30}{\rho}\left(\frac{\|r\|^{2}}{\sqrt{N}}+\left(\mathcal{E}_{N}(r)\right)^{2}\right)^{1 / 2} .
$$

and $C_{1}$ is an absolute constant.

If $\rho=1 / 2$, then Proposition 17 is identical with Proposition 6 in [9]. Moreover, the proof is one and the same for any $\rho>0$ but $\rho$ appears in the formula (6.22). Therefore, we omit the proof of Proposition 17.

Now we complete the proof of (6.3). Lemma 16 together with the inequalities (6.21) and (6.22) in Proposition 17 imply that

$$
\begin{gathered}
A(s) \leq 16 C_{1}(2 C)^{s}\|r\|^{2}\left(1+\|r\|^{2} / a_{N}^{2}\right)(1+s) a_{N}^{2 s} \\
(A(s) A(t))^{1 / 2} \leq 16 C_{1}\|r\|^{2}\left(1+\|r\|^{2} / a_{N}^{2}\right)(1+s)(1+t)\left(2 C a_{N}\right)^{s+t} .
\end{gathered}
$$

By (6.22),$a_{N} \rightarrow 0$ as $N \rightarrow \infty$, so $2 C a_{N}<1$ if $N$ is chosen sufficiently large. Then, the inequality (6.24) guarantees that the series on the right-hand side of (6.7) converges, which implies that (6.3) holds.

Finally, we apply Theorem 2 to the systems of projections

$$
\left\{S_{N}, P_{n, \alpha},|n|>N, \alpha=1,2\right\}, \quad\left\{S_{N}^{0}, P_{n, \alpha}^{0},|n|>N, \alpha=1,2\right\} .
$$

The existence of the Riesz basis $\Phi$ constructed in Lemma 5 implies that the system $\left\{S_{N}^{0}, P_{n, \alpha}^{0},|n|>N, \alpha=1,2\right\}$ is a Riesz basis of projections in $L^{2}\left([0, \pi], \mathbb{C}^{2}\right)$, and by (6.2) and (6.3) the conditions (2.10) and (2.11) are satisfied. Hence, by Theorem 2 , $\left\{S_{N}, P_{n, \alpha},|n|>N, \alpha=1,2\right\}$ is a Riesz basis of projections in $L^{2}\left([0, \pi], \mathbb{C}^{2}\right)$.

Theorem 15 immediately implies the following. 
Corollary 18. The spectrum of $L_{b c}$ is discrete. Each of the discs $D_{n}^{\alpha}, \alpha=1,2, n \in 2 \mathbb{Z},|n|>N$, contains exactly one simple eigenvalue of $L_{b c}$, and the numbers of eigenvalues of $L_{b c}^{0}$ and $L_{b c}$ (counted with their algebraic multiplicity) in $R_{N T}$ are equal, namely

$$
\#\left(S p\left(L_{b c}\right) \cap R_{N T}\right)=\#\left(S p\left(L_{b c}^{0}\right) \cap R_{N T}\right)=2 N .
$$

In view of Corollary [18, the spectrum of the operator $L_{b c}$ could be described by saying that with exception of finitely many points it consists of simple eigenvalues $\lambda_{n, \alpha}$ that are "close" to the corresponding points in the spectrum of the free operator $L_{b c}^{0}$

$$
S p\left(L_{b c}^{0}\right)=\left\{\lambda_{n, \alpha}^{0}=n+\tau_{\alpha}, \quad \alpha=1,2 ; n \in 2 \mathbb{Z}\right\} .
$$

The distance $\left|\lambda_{n, \alpha}-\lambda_{n, \alpha}^{0}\right|$ could be estimate by the norms

$$
\kappa_{n, \alpha}=\left\|P_{n, \alpha}-P_{n, \alpha}^{0}\right\|, \quad n \in 2 \mathbb{Z}, \quad|n|>N, \quad \alpha=1,2,
$$

and the terms $w^{\alpha \alpha}(2 n)$ from the matrix representation of the operator of multiplication $V$ (see Lemma 9). This leads to the following statement.

Theorem 19. In the above notations,

$$
\sum_{|n|>N}\left|\lambda_{n, \alpha}-n-\tau_{\alpha}\right|^{2}<\infty, \quad \alpha=1,2
$$

Proof. Let $\Phi=\left\{\varphi_{n}^{1}, \varphi_{n}^{2}, n \in 2 \mathbb{Z}\right\}$ be the basis of eigenvectors of $L_{b c}^{0}$ constructed in Lemma 5, and let $\left.\tilde{\Phi}=\tilde{\varphi}_{n}^{1}, \tilde{\varphi}_{n}^{2}, n \in 2 \mathbb{Z}\right\}$ be its biorthogonal system. We have

$$
L_{b c}^{0} \varphi_{n}^{\alpha}=\lambda_{n, \alpha}^{0} \varphi_{n}^{\alpha}, \quad P_{n, \alpha}^{0} \varphi_{n}^{\alpha}=\varphi_{n}^{\alpha}, \quad \alpha=1,2 .
$$

and (since $\tilde{\varphi}_{n}^{\alpha}$ are eigenvectors of the adjoint operator $\left(L_{b c}^{0}\right)^{*}$ corresponding to eigenvalues $\overline{\lambda_{n, \alpha}^{0}}$ )

$$
\left(L_{b c}^{0}\right)^{*} \tilde{\varphi}_{n}^{\alpha}=\overline{\lambda_{n, \alpha}^{0}} \tilde{\varphi}_{n}^{\alpha}, \quad \alpha=1,2 .
$$

We set

$$
\psi_{n}^{\alpha}=P_{n, \alpha} \varphi_{n}^{\alpha}, \quad \alpha=1,2 ; \quad n \in 2 \mathbb{Z}, \quad|n|>N .
$$

Then we have

$$
L_{b c} \psi_{n}^{\alpha}=\lambda_{n, \alpha} \psi_{n}^{\alpha}
$$

and

$$
\left\|\psi_{n}^{\alpha}-\varphi_{n}^{\alpha}\right\|=\left\|\left(P_{n, \alpha}-P_{n, \alpha}^{0}\right) \varphi_{n}^{\alpha}\right\| \leq \kappa_{n, \alpha}\left\|\varphi_{n}^{\alpha}\right\| \leq C \kappa_{n, \alpha},
$$

where $C$ is the norm of the isomorphism $A$ from Lemma 5, so $C=$ $C(b c)$. By (6.29),

$$
\lambda_{n, \alpha}\left\langle\psi_{n}^{\alpha}, \tilde{\varphi}_{n}^{\alpha}\right\rangle=\left\langle L_{b c} \psi_{n}^{\alpha}, \tilde{\varphi}_{n}^{\alpha}\right\rangle=\left\langle L_{b c}^{0} \psi_{n}^{\alpha}, \tilde{\varphi}_{n}^{\alpha}\right\rangle+\left\langle V \psi_{n}^{\alpha}, \tilde{\varphi}_{n}^{\alpha}\right\rangle
$$


In view of (6.28),

$$
\left\langle L_{b c}^{0} \psi_{n}^{\alpha}, \tilde{\varphi}_{n}^{\alpha}\right\rangle=\left\langle\psi_{n}^{\alpha},\left(L_{b c}^{0}\right)^{*} \tilde{\varphi}_{n}^{\alpha}\right\rangle=\left\langle\psi_{n}^{\alpha}, \overline{\lambda_{n, \alpha}^{0}} \tilde{\varphi}_{n}^{\alpha}\right\rangle=\lambda_{n, \alpha}^{0}\left\langle\psi_{n}^{\alpha}, \tilde{\varphi}_{n}^{\alpha}\right\rangle .
$$

Therefore, we obtain,

$$
\left(\lambda_{n, \alpha}-\lambda_{n, \alpha}^{0}\right)\left\langle\psi_{n}^{\alpha}, \tilde{\varphi}_{n}^{\alpha}\right\rangle=\left\langle V \psi_{n}^{\alpha}, \tilde{\varphi}_{n}^{\alpha}\right\rangle,
$$

which leads to the formula

$$
\lambda_{n, \alpha}-\lambda_{n, \alpha}^{0}=\frac{\left\langle V \psi_{n}^{\alpha}, \tilde{\varphi}_{n}^{\alpha}\right\rangle}{\left\langle\psi_{n}^{\alpha}, \tilde{\varphi}_{n}^{\alpha}\right\rangle}
$$

By (6.30), it follows that

$$
\left\langle\psi_{n}^{\alpha}, \tilde{\varphi}_{n}^{\alpha}\right\rangle=\left\langle\varphi_{n}^{\alpha}, \tilde{\varphi}_{n}^{\alpha}\right\rangle+\left\langle\left(\psi_{n}^{\alpha}-\varphi_{n}^{\alpha}\right), \tilde{\varphi}_{n}^{\alpha}\right\rangle=1+O\left(\kappa_{n, \alpha}\right) .
$$

On the other hand,

$$
\left\langle V \psi_{n}^{\alpha}, \tilde{\varphi}_{n}^{\alpha}\right\rangle=\left\langle V \varphi_{n}^{\alpha}, \tilde{\varphi}_{n}^{\alpha}\right\rangle+\left\langle V\left(\psi_{n}^{\alpha}-\varphi_{n}^{\alpha}\right), \tilde{\varphi}_{n}^{\alpha}\right\rangle .
$$

By Lemma 9 and (4.3), we have

$$
\left\langle V \varphi_{n}^{\alpha}, \tilde{\varphi}_{n}^{\alpha}\right\rangle=w^{\alpha \alpha}(2 n), \quad \text { where }\left.\quad \sum_{n} w^{\alpha \alpha}(2 n)\right|^{2}<\infty .
$$

In view of (6.30),

$$
\left\langle V\left(\psi_{n}^{\alpha}-\varphi_{n}^{\alpha}\right), \tilde{\varphi}_{n}^{\alpha}\right\rangle=\left\langle\left(\psi_{n}^{\alpha}-\varphi_{n}^{\alpha}, V^{*} \tilde{\varphi}_{n}^{\alpha}\right\rangle=O\left(\left\|\psi_{n}^{\alpha}-\varphi_{n}^{\alpha}\right\|\right)=O\left(\kappa_{n}\right)\right.
$$

because the functions $\tilde{\varphi}_{n}^{\alpha}$ are uniformly bounded due to Lemma 5 , Formulas (3.26) and (3.27).

Therefore, by (6.31), we obtain

$$
\lambda_{n, \alpha}-\lambda_{n, \alpha}^{0}=\frac{w^{\alpha \alpha}(2 n)+O\left(\kappa_{n}\right)}{1+O\left(\kappa_{n}\right)},
$$

From here (6.27) follows because $\sum_{n}\left|w^{\alpha \alpha}(2 n)\right|^{2}<\infty$ by (4.3) and $\sum_{n} \kappa_{n}^{2}<\infty$ by (6.3) .

\section{BARI-MARKus PROPERTY IN THE CASE OF REGUlAR BUt NOT STRICTLY REGULAR BOUNDARY CONDITIONS}

We use the notations of Section 5. For regular but not strictly regular $b c$ Theorem 14 gives the following localization of the spectrum of the Dirac operator $L_{b c}$ :

$$
S p\left(L_{b c}\right) \subset R_{N T} \cup \bigcup_{|n|>N} D_{n} .
$$

Let us consider the Riesz projections associated with $L_{b c}$

$$
S_{N}=\frac{1}{2 \pi i} \int_{\partial R_{N T}}(\lambda-L)^{-1} d \lambda, \quad P_{n}=\frac{1}{2 \pi i} \int_{\partial D_{n}}(\lambda-L)^{-1} d \lambda,
$$


and let $S_{N}^{0}$ and $P_{n}^{0}$ be the Riesz projections associated with the free operator $L_{b c}^{0}$.

Theorem 20. Suppose $L_{b c}$ and $L_{b c}^{0}$ are, respectively, the Dirac operator with an $L^{2}$ potential $v$ and the corresponding free Dirac operator, subject to regular but not strictly regular boundary conditions bc. Then, there is an $N \in 2 \mathbb{N}$ such that the Riesz projections $S_{N}, P_{n}$ and $S_{N}^{0}, P_{n}^{0}, n \in$ $2 \mathbb{Z},|n|>N$, associated with $L$ and $L^{0}$ are well defined by (6.1), and we have

$$
\begin{gathered}
\operatorname{dim} P_{n}=\operatorname{dim} P_{n}^{0}=2, \quad \operatorname{dim} S_{N}=\operatorname{dim} S_{N}^{0}=2 N ; \\
\sum_{|n|>N}\left\|P_{n}-P_{n}^{0}\right\|^{2}<\infty .
\end{gathered}
$$

Moreover, the system $\left\{S_{N} ; P_{n}, n \in 2 \mathbb{Z},|n|>N\right\}$ is a Riesz basis of projections in $L^{2}\left([0, \pi], \mathbb{C}^{2}\right)$, i.e.,

$$
\mathbf{f}=S_{N}(\mathbf{f})+\sum_{|n|>N} P_{n}(\mathbf{f}) \quad \forall \mathbf{f} \in L^{2}\left([0, \pi], \mathbb{C}^{2}\right),
$$

where the series converge unconditionally.

Proof. One may prove the theorem by repeating (with a few obvious adjustments) the proof of Theorem 15. Therefore, the proof is omitted.

Theorem 20 immediately implies the following.

Corollary 21. The spectrum of $L_{b c}$ is discrete. Each of the discs $D_{n}, n \in 2 \mathbb{Z},|n|>N$, contains exactly two eigenvalues (counted with algebraic multiplicity) of $L_{b c}$, and the numbers of eigenvalues of $L_{b c}^{0}$ and $L_{b c}$ (counted with algebraic multiplicity) in $R_{N T}$ are equal, namely

$$
\#\left(S p\left(L_{b c}\right) \cap R_{N T}\right)=\#\left(S p\left(L_{b c}^{0}\right) \cap R_{N T}\right)=2 N .
$$

\section{Miscellaneous; POINTWise COnVERGence AND EQUICONVERGENCE}

1. Suppose that $L_{b c}^{0}$ is the free Dirac operator considered with regular boundary conditions $(b c)$ given by the matrix $\left[\begin{array}{llll}1 & b & a & 0 \\ 0 & d & c & 1\end{array}\right]$ in (3.11). Let $\Phi=\left\{\varphi_{k}^{1}, \varphi_{k}^{2}, k \in \mathbb{Z}\right\}$ be the corresponding Riesz basis in $L^{2}\left([0, \pi], \mathbb{C}^{2}\right)$ consisting of eigenfunctions and associated functions of $L_{b c}^{0}$, which is constructed, respectively, in Lemma 5 if $b c$ is strictly regular, in Lemma 7 if $b c$ is periodic type, and in Lemma 8 otherwise. 
Then we have

$$
\sum_{m \in 2 \mathbb{Z}} \sum_{\mu=1}^{2}\left\langle\left(\begin{array}{l}
f \\
g
\end{array}\right), \tilde{\varphi}_{m}^{\mu}\right\rangle \varphi_{m}^{\mu}=\left(\begin{array}{l}
f \\
g
\end{array}\right), \quad \forall f, g \in L^{2}([0, \pi], \mathbb{C}),
$$

where the series converges unconditionally in $L^{2}\left([0, \pi], \mathbb{C}^{2}\right)$. The following statement gives sufficient conditions for point-wise convergence of the series in (8.1) and explains what is its sum for each $x \in[0, \pi]$.

Pointwise Convergence Theorem. If $f, g:[0, \pi] \rightarrow \mathbb{C}$ are functions of bounded variation which are continuous at 0 and $\pi$, then

$$
\lim _{M \rightarrow \infty} \sum_{m=-M}^{M}\left[\left\langle\left(\begin{array}{l}
f \\
g
\end{array}\right), \tilde{\varphi}_{m}^{1}\right\rangle \varphi_{m}^{1}(x)+\left\langle\left(\begin{array}{l}
f \\
g
\end{array}\right), \tilde{\varphi}_{m}^{2}\right\rangle \varphi_{m}^{2}(x)\right]=\left(\begin{array}{c}
\tilde{f}(x) \\
\tilde{g}(x)
\end{array}\right)
$$

where

$$
\left(\begin{array}{c}
\tilde{f}(x) \\
\tilde{g}(x)
\end{array}\right)=\frac{1}{2}\left(\begin{array}{c}
f(x-0)+f(x+0) \\
g(x-0)+g(x+0)
\end{array}\right) \quad \text { for } x \in(0, \pi)
$$

and

$$
\left(\begin{array}{l}
\tilde{f}(x) \\
\tilde{g}(x)
\end{array}\right)= \begin{cases}\frac{1}{2}\left(\begin{array}{c}
f(0)-b f(\pi)-a g(0) \\
\frac{d}{b c-a d} f(0)+g(0)-\frac{b}{b c-a d} g(\pi)
\end{array}\right) & \text { if } x=0, \\
\frac{1}{2}\left(\begin{array}{c}
-\frac{c}{b c-a d} f(0)+f(\pi)+\frac{a}{b c-a d} g(\pi) \\
-d f(\pi)-c g(0)+g(\pi)
\end{array}\right) & \text { if } x=\pi .\end{cases}
$$

Moreover, if both $f$ and $g$ are continuous on some closed subinterval of $(0, \pi)$ then the convergence (8.2) is uniform on that interval.

2. Next, suppose that $v$ is an $L^{2}([0, \pi])$ Dirac potential and consider the operator $L_{b c}(v)$.

For strictly regular $b c$, Theorem 15] shows that there is a Riesz basis of projections; Formula (6.4) is an analog of (8.1). Moreover, since the projections $P_{n}^{\alpha}$ that appear in (6.4) are one-dimensional while $\operatorname{dim} S_{N}=$ $2 N$, in fact Theorem 15 proves the existence of a Riesz basis $\varphi_{m}^{\mu}, m \in$ $2 \mathbb{Z}, \mu \in\{1,2\}$, consisting of eigenfunctions and at most finitely many associated functions of the operator $L_{b c}(v)$.

For regular but not strictly regular $b c$, the existence of Riesz basis of projections is proven in Theorem 20, see Formula (7.4). The Riesz projections $P_{m}$ that appear in (7.4) are two-dimensional, and in general it is impossible to "split" the corresponding two-dimensional subspaces into one-dimensional so that to get a Riesz basis of functions (see in [10] results about existence and nonexistence of Riesz basis of functions in the case of periodic or antiperiodic $b c$ ). 
However, in both cases the spectral decompositions (8.1)) of $L_{b c}^{0}$ and the spectral decompositions of $L_{b c}(v)$ given, respectively, by (6.4) for strictly regular $b c$ and by (7.4) for regular but not strictly regular $b c$, converge pointwise to the same limit, or diverge simultaneously, due to the following.

Equiconvergence Theorem. Let $S_{N}=S_{N}(v, b c)$ and $S_{N}^{0}(b c)$ be the projections defined by (6.1), and let $F:[0, \pi] \rightarrow \mathbb{C}$ be a function of bounded variation. Then, for every regular bc and every $L^{2}([0, \pi])$ potential $v$,

$$
\left\|\left(S_{N}-S_{N}^{0}\right) F\right\|_{\infty} \rightarrow 0 \quad \text { as } \quad N \rightarrow \infty
$$

Proofs and generalizations of these results will be presented elsewhere. We are thankful to R. Szmytkowski for bringing our attention to the point-wise convergence problem of spectral decompositions of 1D Dirac operators. In the case of separated boundary conditions, our point-wise convergence results confirm the formula suggested by R. Szmytkowski ([25, Formula 3.14]).

\section{REFERENCES}

[1] Bari, N. K. Biorthogonal systems and bases in Hilbert space. (Russian) Moskov. Gos. Univ. U čenye Zapiski Matematika 148(4), (1951). 69-107.

[2] G. D. Birkhoff, On the asymptotic character of the solutions of certain linear differential equations containing a parameter, Trans. Amer. Math. Soc. 9 (1908), 21-231.

[3] G. D. Birkhoff, Boundary value and expansion problems of ordinary linear differential equations, Trans. Amer. Math. Soc. 9 (1908), 373-395.

[4] P. Djakov and B. Mityagin, Smoothness of Schrödinger operator potential in the case of Gevrey type asymptotics of the gaps, J. Funct. Anal. 195 (2002), 89-128.

[5] P. Djakov and B. Mityagin, Spectral triangles of Schrödinger operators with complex potentials. Selecta Math. (N.S.) 9 (2003), 495-528.

[6] P. Djakov and B. Mityagin, Instability zones of a periodic 1D Dirac operator and smoothness of its potential. Comm. Math. Phys. 259 (2005), 139-183.

[7] P. Djakov and B. Mityagin, Spectra of 1-D periodic Dirac operators and smoothness of potentials. C. R. Math. Acad. Sci. Soc. R. Can. 25 (2003), $121-125$.

[8] P. Djakov and B. Mityagin, Instability zones of periodic 1D Schrödinger and Dirac operators (Russian), Uspehi Mat. Nauk 61 (2006), no 4, 77-182 (English: Russian Math. Surveys 61 (2006), no 4, 663-766).

[9] P. Djakov and B. Mityagin, Bari-Markus property for Riesz projections of 1D periodic Dirac operators, Math. Nachr. 283 (2010), no. 3, 443-462.

[10] P. Djakov and B. Mityagin, 1D Dirac operators with special periodic potentials, arXiv:1007.3234 
[11] N. Dunford, A survey of the theory of spectral operators, Bull. Amer. Math. Soc. 64 (1058), $217-274$.

[12] N. Dunford, J. Schwartz, Linear Operators, Part III, Spectral Operators, Wiley, New York, 1971.

[13] I. C. Gohberg, M. G. Krein, Introduction to the theory of linear non-selfadjoint operators, vol. 18 (Translation of Mathematical Monographs). Providence, Rhode Island, American Mathematical Society 1969.

[14] S. Hassi and L. Oridoroga, Theorem of Completeness for a Dirac-Type Operator with Generalized $\lambda$-Depending Boundary Conditions, Integral Equat. Oper. Theor. 64 (2009), 357-379.

[15] G. M. Keselman, On the unconditional convergence of eigenfunction expansions of certain differential operators, Izv. Vyssh. Uchebn. Zaved. Mat. 39 (2) (1964), 82-93 (Russian).

[16] M. M. Malamud and L. L. Oridoroga, Theorems of the Completeness for the Systems of Ordinary Differential Equations, Functional Analysis and Applications 34, No 3, (2000), 88-90.

[17] Markus, A. S. A basis of root vectors of a dissipative operator. Dokl. Akad. Nauk SSSR 132 524-527 (Russian); translated as Soviet Math. Dokl. 11960 599-602.

[18] V. P. Mikhailov, On Riesz bases in $L^{2}(0,1)$, Dokl. Akad. Nauk SSSR 144 (1962), 981-984 (Russian).

[19] B. Mityagin, Convergence of expansions in eigenfunctions of the Dirac operator. (Russian) Dokl. Akad. Nauk 393 (2003), no. 4, 456-459.

[20] B. Mityagin, Spectral expansions of one-dimensional periodic Dirac operators. Dyn. Partial Differ. Equ. 1 (2004), 125-191.

[21] M. Reed and B. Simon, Methods of modern mathematical physics, vol. I, Academic Press, New York, 1975.

[22] A. A. Shkalikov, The basis property of eigenfunctions of an ordinary differential operator, Uspekhi Mat, Nauk 34 (1979), 235 - 236 (Russian)

[23] A. A. Shkalikov, On the basisness property of eigenfunctions of ordinary differential operators with integral boundary conditions, Vestnik Mosk. Univ., ser. 1, Math. \& Mech., 6 (1982), $12-21$.

[24] A. A. Shkalikov, Boundary value problems for ordinary differential equations with a parameter in the boundary conditions, Trudy Sem. I. G. Petrovskogo 9 (1983), 190 - 229 (Russian); English transl.: J. Sov. Math. 33 (6) (1986), 1311 $-1342$

[25] R. Szmytkowski, Discontinuities in Dirac eigenfunction expansions, J. Math. Physics 42, 4606-4617.

[26] J. D. Tamarkin, Sur quelques points de la theorie des equations di?erentielles lineaires ordinaires et sur la generalisation de la serie de Fourier, Rend. Circ. Mat. Palermo (2) 34 (1912), 345-382.

[27] J. D. Tamarkin, On some general problems of the theory of ordinary linear differential operators and on expansion of arbitrary function into serii, Petrograd. 1917, $308 \mathrm{p}$.

[28] J. D. Tamarkin, Some general problems of the theory of linear di?erential equations and expansions of an arbitrary functions in series of fundamental functions, Math. Z. 27 (1928), 1-54. 
[29] I. Trooshin and M. Yamamoto, Riesz basis of root vectors of a nonsymmetric system of first-order ordinary differential operators and application to inverse eigenvalue problems, Appl. Anal. 80, (2001), 19-51.

[30] I. Trooshin and M. Yamamoto, Spectral properties and an inverse eigenvalue problem for nonsymmetric systems of ordinary differential operators, J. Inverse Ill-Posed Probl. 10 No 6, (2002), 643-658.

Sabanci University, Orhanli, 34956 Tuzla, Istanbul, Turkey

E-mail address: djakov@sabanciuniv.edu

Department of Mathematics, The Ohio State University, 231 West 18Th Ave, Columbus, OH 43210, USA

E-mail address: mityagin.1@osu.edu 